SF 263

.58

Copy 1

\title{
HE LECITHIN CONTENT OF BUTTER AND ITS POSSIBLE RELATIONSHIP TO THE FISHY FLAVOR
}

\author{
A THESIS \\ Presented to the Faculty of the Graduate School \\ of Cornell University for the Degree of \\ DOCTOR OF PHILOSOPHY
}

BY

GEORGE CORNELL SUPPLEE 



\title{
THE LECITHIN CONTENT OF BUTTER AND ITS POSSIBLE RELATIONSHIP TO THE FISHY FLAVOR
}

\author{
A THESIS \\ Presented to the Faculty of the Graduate School \\ of Cornell University for the Degree of \\ DOCTOR OF PHILOSOPHY
}

BY

GEORGE CORNELL SUPPLEE 


$$
\begin{array}{r}
58+3 \\
58
\end{array}
$$

5. OL

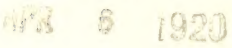




\section{CONTENTS}

PAGE

Previous investigations. . . . . . . . . . . . . . . . . . . . 101

Lecithin decomposition in butter as a possible cause of the fishy flavor 104

Chemical constitution, properties, and distribution of lecithin... 104

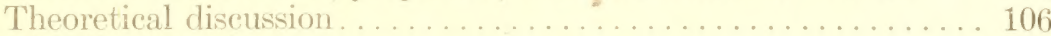

Investigational work. . . . . . . . . . . . . . . . . . . . . . . . 108

Qualitative determination of lecithin in butter............. 108

Amount of lecithin in butter....................... 110

Trimethylamine salts of the fatty acids................ 111

Effect of working trimethylamine salts of the fatty acids into

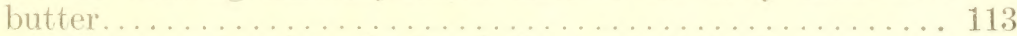

Quantitative estimation of trimethylamine in fishy butter.... 123

Development of fishy flavor in experimental butters.......... 126

Variation in acid value of experimental butters............. 129

Trimethylamine and ammonia content of experimental butters. 134

Bacteriological studies. . . . . . . . . . . . . . . . . . . 136

Bacterial analysis......................... 137

Inoculations for the purpose of developing the fishy flavor. . 137

Longevity of Bacterium ichthyosmius in butter............... 141

Further studies with Bacterium ichthyosmius.............. 142

Trimethylamine and ammonia production by Bacterium ich-

thyosmius in milk and cream....................... 144

Production of trimethylamine from lecithin and choline by

bacterial action. . . . . . . . . . . . . . . . . . . . 145

Bacterial inoculations into butterfat. ............... 146

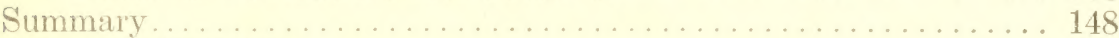

Acknowledgments.................................. 149

Bibliography. . . . . . . . . . . . . . . . . . . . . . 150 
THE LECITHIN CONTENT OF BUTTER AND ITS POSSIBLE RELATIONSHIP TO THE FISHY FLAVOR 



\section{THE LECITHIN CONTENT OF BUTTER}

\section{AND ITS POSSIBLE RELATIONSHIP TO THE FISHY FLAVOR ${ }^{1}$ \\ George Cornell Supplee}

The commercial value of butter is based to a great extent on its quality, which in turn is determined by its flavor. The commercial grading of butter on this basis, and the recognition of certain characteristic defects, have resulted in the establishment of certain terms more or less descriptive of the flavors found. Among the terms commonly applied to the flavors in butter are such words as metallic, fishy, oily, rancid, tallowy. Since the presence of any of these flavors carries with it a reduction in commercial value of the butter, considerable effort has been made to determine their causes and prevent their development. Unfortunately most of these efforts have not met with a high degree of success. This may be ascribed to several reasons, among which are the following: lack of positive identification of the same flavor by different investigators; lack of adequate chemical methods for the isolation and measurement of the small amount of substance capable of producing the flavor; and lack of cooperation between the chemist, the bacteriologist, and the experienced butter judge.

Fishiness in butter, with which this investigation is primarily concerned, is usually described as a flavor resembling that of salmon or mackerel, altho the names of other varieties of fish are occasionally used to describe the flavor more explicitly. While the typical fishy flavor in butter is readily recognized by experts, it is often accompanied by a more or less oily condition which tends to create differences of opinion as to its exact nature. But if the opinion of butter judges of long experience is to be considered as trustworthy, it may be said that the true fishy flavor is entirely distinet from the oily flavor even tho the oily condition may precede or accompany it.

\section{PREVIOUS INVESTIGATIONS}

The earlier investigations bearing on fishiness in dairy products have been largely confined to milk and butter. One instance is recorded, however, in which this condition was observed and studied in evaporated milk.

\footnotetext{
1 Also presented to the Faculty of the Graduate School of Cornell University, December, 1918, as a major thesis in partial fulfillment of the requirements for the degree of doctor of philosophy.
} 
Harding, Rogers, and Smith $(1900)^{2}$ report the fishy flavor in a sample of milk brought to the New York State Agricultural Experiment Station at Geneva in 1900. The source was traced to a single animal in the herd, but investigation failed to locate any pathological condition or any irregularity in the feeding which might cause the trouble. Attempts to reproduce the flavor by inoculating milk with bacteria isolated from this cow's udder also failed. The same authors mention also an instance brought to their attention by W. E. Griffith, in which a peculiar flavor developed in June butter after storage at $18^{\circ}$ to $22^{\circ} \mathrm{F}$. This flavor was deseribed by butter experts as fishy.

Piffard (1901) discusses the fishy flavor in dairy products, and suggests the possible relationship between certain algae found in stagnant water, and fishiness in milk and butter. His theory is supported by the fact that the flavor is often produced in water by the growth of algae and diatoms, and he believes, therefore, that cows having access to such water may transmit the condition to milk. Referring to the flavor in butter, he considers the idea that salt may be responsible and mentions the ability of salt to absorb flavors and odors of materials stored near it.

Harrison (1902), discussing butter defects at about the same time, states that the characteristic off-flavors of butter - fishy butter being specifically mentioned - are caused by the growth of undesirable bacteria in the cream.

O'Callaghan (1902) published certain observations on fishiness in Australian butter. He states that he has found this condition in butter only two hours old. From his investigations he concludes that Oidium lactis is the causal agent. Later (O'Callaghan, 1908) he elaborated on his former views, concluding that Oidium lactis associated with the lacticacid bacteria in cream will usually produce a fishy flavor in the butter. He mentions the presence of the defect in unsalted butter, and recommends the improvement of sanitary conditions in the creameries, and pasteurization, as a remedy. His conclusions have not been confirmed by other investigators.

Rogers (1909), after a rather exhaustive study of the occurrence and cause of fishy butter, confirms many observations commonly noted in connection with this trouble but is unable to confirm the observations of O'Callaghan. He also ssemingly eliminates the theory that trimethyl-

2 Dates in parenthesis refer to Bibliography, page 150 . 
amine is directly responsible for the flaror. After studying the effect of high-acid cream, overworking, and the consequent increased oxygen content of the butter, and by conducting bacteriological investigations, he concludes that high-acid cream is essential to bring about the condition, altho he points out that not all butter made from such cream develops the fishy flavor. In this respect he states (page 20 of reference eited) that "fishy flavor may be produced with reasonalle certainty by overworking the butter made from sour ercam." From his viewpoint the probability that microorganisms are the cause falls into disfaror by the advancement of the opinion that "fishy flaror is caused by a slow, spontaneous, chemical change to which aciel is essential and which is favored by the presence of small amounts of oxygen" (page 20 of reference). More recently the same aluthor. (Rogers, 1914, a and b) points out that fishiness in butter may be preceder by an oily or a metallic flavor, and reiterates his views that the eridence is against the theory that the fishy condition is of a bacterial nature. He also states (1914b) that "fishy" flavor is said to oceur rarely or not at all in unsalted butter and it is possible that the salt furnishes eertain conditions which are essential to the development of the flavor."

Reakes, Cuddie, and Rach (19)12) find no significant differenees in the bacterial flora of fishy and of high-orade butter, and, in agreement with Rogers, state that "the development of fishy flavour in butter arises as a result of a chemieal change indueing a splitting-up of some of the constituents into compound posmesing this peculiar character of smell and taste, the factors responsible for such change being apparently a degree of high acidity of the "ream and overworking."

Hunziker (1916) states that high pasteurization temperatures $\left(185^{\circ} \mathrm{F}\right.$.) when used on sour cream tend to produce at rery poor quality of butter, which often has a disagrecalile oily taste suggestive of fishiness. He points out that this is particularly true when cows are on green pasture and the butterfat contains a rather high perecntage of olein, which may ba oxidized with relative ase in the presence of high temperatures and high acid.

Hammer (1917) reports that he foumel at can of evaporated milk which possessed a marked fishy flavor and odtor and from which he was able to isolate an organism heretofores undescriberl. He gives to this organism the name Bacterium ichthyosmius, which was suggested by Dr. A. IV. 
Dox. The deseription of the organism seems to indicate that it is closely allied to the Proteus group. By inoculation experiments Hammer was able to reproduce the flavor in milk and cream under both aerobic and anaerobic conditions. He noted that the intensity of the odor was increased by the addition of alkali to the milk after the incubation period. He was unable, however, to produce fishiness in butter by direct inoculation or by inoculating the cream before churning. Bacteria counts at various intervals during the storage period showed an immediate decrease in numbers in salted butter, and an increase during the first few days in unsalted butter followed by a pronounced decrease.

Washburn and Dahlberg (1918), while studying the influence of salt on storage butter, found that salted butter was more likely to turn fishy in storage than was unsalted butter, and furthemore that there appeared to be a tendency toward a progressive development of the flavor thru metallic to oily and finally to fishy.

\section{LECITHIN DECOAPOSITION IN BUTTER AS A POSSIBLE CAUSE OF THE FISIIY FLAVOR}

CHEMICAL CONSTITUTION, PROPERTIES, AND DISTRIBUTION OF LECITHIN

Lecithin, which stands in close relation to the fats, belongs to a more or less definite group of substances known as phosphlatides, or phosphorized fats. 'These bodies appear to be a group of ester's containing nitrogen, phosphorie acid, and fatty-acid radicals. Lecithin, which is the best known of the phosphatides, contains two fatty-acid radicals and the nitrogenous base choline, combined with glyeerophosphoric acir. Aceording to the kind of fatty acid present in the molecule, it is possible to have various types of lecithin, such as stearyl, palmityl, and oleyl. A number of investigators secm to agree that every true lecithin contains at least one oleic-acid radieal. There seems to be unecrtainty as to whether choline is the only base present in lecithin. MacLean (1909) was able to get only t'2 per cent of the theoretical amount from lecithin isolated from heart muscle, and (5.5 per cent from lecithin of ego yolk. Other investigators have found the same to hold true of lecithin from different sources.

Lecithin has certain properties in common with the fats, particularly with respect, to its solvents. It differs, however, by being less soluble 
in ether and more soluble in alcohol. It is precipitated from alcoholic solution by acetone; in water it swells to a colloidal mass which on microscopic examination appears as oily drops and threads. It saponifies with alkalies and baryta water, yielding the corresponding soaps, salts of glycerophosphoric acid, and choline. Hammarsten and Hedin (1915) state that it is slowly decomposed by dilute acids and enzymes (lipase). Barger (1914) states that Bacterium prodigiosus produces trimethylamine from choline and lecithin; he also cites references to show that lecithin is decomposed during putrefaction, yielding fatty acids, glycerophosphoric acid, choline, and ultimately trimethylamine. Hasebroek (1888) claims that methylamine, ammonia, methane, and carbon dioxide may be finally produced from choline during putrefaction. On being heated with strong caustic soda or potash, lecithin yields trimethylamine, which has a distinct fishy odor, this being one of the characteristic qualitative tests for it. Leathes (1913), in citing the work of various investigators, seems to think that lecithin is rather unstable. He thinks this property is due to the unsaturated oleic-acid radical contained, and offers this as the reason why the substanee gives Pettenkofer's reaction. The work of Long (1908), howerer, seems to indicate that lecithin is more stable than has been generally believed. Koch (1902-03) has shown that various salts will cause lecithin to precipitate as a gelatinous mass, and that acids, if sufficiently dissociated (0.005 M sulfuric), will accomplish the same thing.

Lecithin secms to be widcly found in nature, being present in many plant cells and animal fluids. It is particularly abundant in the brain, the nerve tissue, and the yolk of egg. It is also reported as existing in blood corpuseles, blood plasma, lymph, milk, and bile. Since the methods used for the quantitative crtimation of lecithin depend on the determination of phosphorus in alcoholic or alcohol and ether extracts, it is doubtful whether the figures given are absolutcly correct due to the faet that other phosphatides are extracted and also because the empirical formula used in the calculation may be inaceurate for the particular lecithin involved. Altho there have been conflicting statements as to whether milk contains lecithin, there seems to be sufficient evidence that it does. The results obtained by Nerking and Haensel (1908) are submitted in table 1: 
TABLE 1. Tire Lecithin Content of Vanotes Miliks (From Nerking and Harensel)

\begin{tabular}{|c|c|c|c|}
\hline \multirow{2}{*}{ Kind of milk } & \multicolumn{3}{|c|}{ l'ercentage of lecithin } \\
\hline & Highest & Lowest & Average \\
\hline 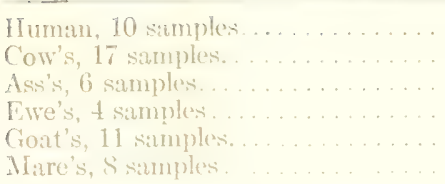 & $\begin{array}{l}0.080 \\
0.1117 \\
0.039 \\
0.117 \\
0.075 \\
0.017\end{array}$ & $\begin{array}{l}0.024 \\
0.036 \\
0.006 \\
0.051 \\
0.036 \\
0.007\end{array}$ & $\begin{array}{l}0.050 \\
0.0633 \\
0.016 \\
0.0853 \\
0.049 \\
0.011\end{array}$ \\
\hline
\end{tabular}

Glikin (1909), sturying the lecithin and iron content of milk, reports 0.0515 per "ent levithin in whole milk, 0.0.) pere cont in cream, and 0.1329 per cent in human milk. Frotzor (1911), studying the lecithin content of milk mulkr palluologioal anditions, finds that it, is lower in milk from (rows suffering with mistitis than in mitk from nommal cows. He finds

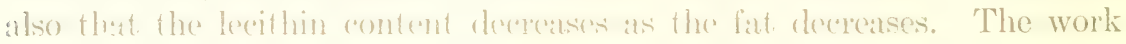

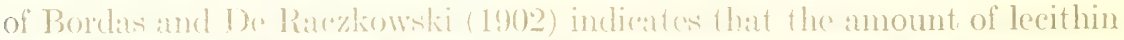
values with the laretation poriod. They find that it is at the maximum

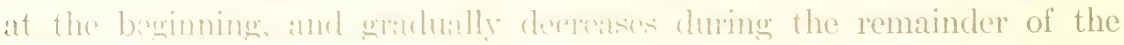

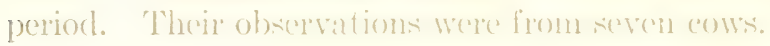

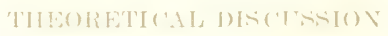

Trimethylamine as a deremposition proment of lecithin was brought to the attertion of the writer ats a possible catuse of the fishy flavor in

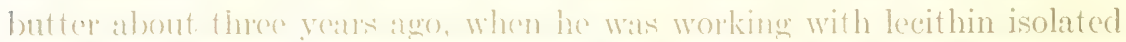

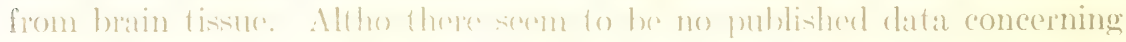

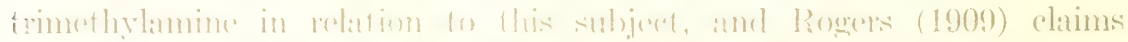

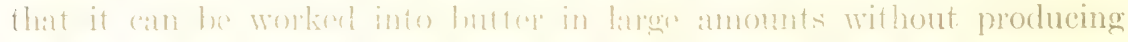

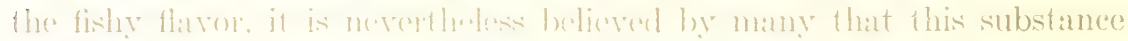

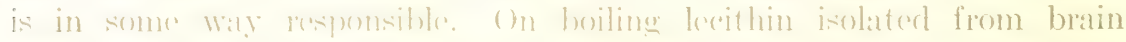

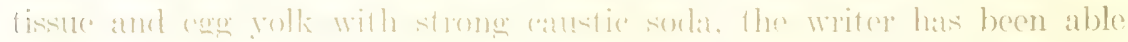

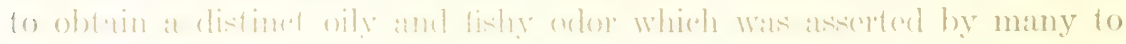

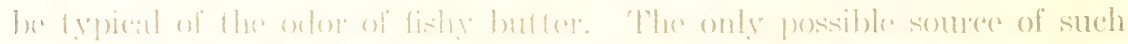

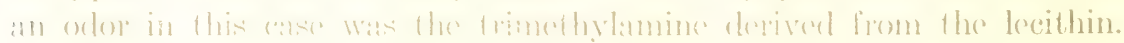

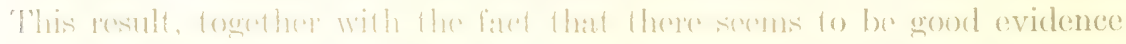
that lecithin is peresent in milli. led to the assmention that the substance 
may exist also in butter and that by its decomposition it can slowly liberate trimethylamine with the consequent production of the fishy flavor and odor.

Provided that lecithin can be shown to exist in butter, the above assumption is supported by several facts. It is well known that enzymes are capable of bringing about many chemical decompositions which result from the action of acids and alkalies on organic substances. It also appears to be a fact that butter may contain enzymes derived from the udder, and from the action of bacteria in the milk or the cream before it is made into butter, and furthermore that the activity of such enzymes is not entirely stopped at the temperature at which butter is stored. Hammarsten and Hedin (1915) state that lecithin is decomposed by dilute acids and enzymes. The citations of Hasebrock (1888), Barger (1914), and others show that lecithin is decomposed by bacteria yielding choline, which finally yields trimethylamine. It is also a well-known fact that this substance in very small amounts possesses a distinet fishy odor, but in concentrated solution it has a strong ammoniacal odor. Speaking of the former property, Davis (1912), quoting Taylor, states that the "odour [referring to the peculiar fishy odor suggestive of herring brine] is gradually developed by adding lime to a solution of the base, but requires some time to reach its maximum intensity."

In addition to the foregoing facts the writer has observed certain features that may have some bearing on this problem. In inspecting butter used in the Navy, it has been noticed that certain samples of cream evolved a peculiar fishy odlor on the addition of alkali used for titration. This phenomenon was first brought to the attention of the writer by A. M. Besemer, and has since been confirmed by a number of men, some of whom have wide reputation as butter judges. Since trimethylamine is a base which is liberated from its acid combinations by alkalies, it is quite possible that the odor mentioned above was due to this substance's having been liberated from its acid combination in the cream. If such were the case, it is conceivable that buttcr made from such cream might, during storage, give up its trimethylamine thru the action of enzymes. In this connection it has been noted that certain samples of old butter, which were not scored as fishy, when brought into contact with a warm solution of soap powder would give off a strong herring-like odor. This phenomenon might also be explained as in the case of the cream. In addition to these features it has been noted that certain samples of fishy 
butter may lose their characteristie flavor after a period of time. This has also been oberred by oflere investigators. It is possible that this characteristic may be explained by the fact that, sinee trimethylamine is extremely volatile, it may pass off, or that the instability of the acid combination changes so that the eonditions are not right for its manifestation. The writer has noted a very strong fishy odor in partially decomposed cog yolk held at lefrigerator temperature, which had entirely disappeared two weeks later.

On the basis of the foregoing facts and observations and the evident lack of contradiction of most of them with what is known about fishy butter, the following experimental work was plamed with the object of dotermining the posible relationship of trinethylamine to this flavor. In ealling attention to the leceithin, it may be stated that the writer is cognizant of the faret that trimethylamine may be produced from other substanees. This material has been chosen as the object of study primarily berause there is cxact knowledge concerning its eleavage and some of the ageneies bringing this condition about.

\section{INVHTTETIONAL WOHK}

QTALTATITE IOETERTINATION OF LE(ITHIN IN BUTTER

The first experimental work undertaken was to demonstrate the presenere of leceithin in butere, sinere there appeared to be no reports on this print in the literature. ( ne humbered gramis of melted butter was thoroly mixed with suffieimt anhydrons caleimm sulfate (about one kilogram) so that the mixture retained its fry powdered form to such an extent that it andel be readily siffed betwern the thumb and the finger. The mixture was transtered to al specially ronstructerl pereolator and extracted

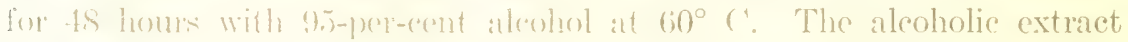
was craporated form and the residue was treated with a small amount of othep, which took up the hat, the fatty arcids, and part of the lecithin. The part insoluble in ethere was atgin faken up with wam alcohol, and what may be termed the lecithin frection was presipitated by thoroly ronling the alcoholis solution. The substanes thus obtained precipitated in the form of small, wart-like masses, which clung tenaciously to the sirtes of the beakire. (On this material, which presumably contained a high propention of leceithin, rarions observations were made and qualitafive tests alpm)lied. 'The following chatacteristices were noted: 
On drying at ordinary temperature and pressure the material appeared as a semi-amorphous and oily substance of a pale, dirty yellow color. It was entirely soluble in alcohol but was partially thrown out of solution by the addition of an excess of ether. The precipitate formed in this manner was finely granular and was white in color. In water it formed a semi-colloidal solution which on microscopic examination appeared as minute oily drops. When the watery suspension was heated, the particles would cohcre to form a sticky mass which changed to a distinct brown color. Both the dry substance and the watery suspension, when heated with strong caustic soda, gave off a marked fishy odor resembling sometimes dried herring and sometimes salmon oil. This observation was in the great majority of eases confurmed by a number of colleagues. The fishy odor' obtained from the material in this manner seemed to furnish positive evidence that lecithin was present. To further strengthen this belief, Pettenkofer's test with sugar and sulfuric acid was applied to the dry material with positive results. The above observations were confirmed with lecithin extracted from fresh butter, salted and unsalted, and from other miscellancous samples of normal butter.

Altho the evidence that lecithin exists in butter in detectable quantities seemed conclusive, it was decided to determine, if possible, the presence of choline, which, as already pointed out, is one of the components of the lecithin molecule. This was accomplished by boiling the residue of the first alcoholic extract with baryta water, which removed all fat, fatty acids, and fatty-acid radieals of the lecithin in the form of barium soaps. After the barium soaps were filtered off, the excess barium was removed with carbon dioxide, the barim carbonate filtered off, and the filtrate containing choline and barim glycerophosphate evaporated to a sirupy consistency. This residue was then treated with absolute alcohol, in which choline is soluble but barium glycerophosphate is insoluble. On evaporation of the alosolute alcohol a small amount of sirupy substance remained. To this matcrial qualitative tests for choline were applied. The most characteristic of such tests is the periodide test described by Stanĕk (1905), which is made by adding a small amount of strong iodine solution (153 grams of iodine and 100 grams of potassium iodide in 200 grams of water) to an aqueous solution of choline. A positive test is indicated by the formation of a brown precipitate of choline periodide, which on mieroscopic examination in the presence of the reagent appears as dark brown refractive and notehed prisms or rhomboidal 
leaflets. On evaporation of the reagent the erystals lose their shape and appear to liquefy, forming brown, oily droplets which again assume their erystalline structure on the addition of more reagent. On the application of this test to the choline obtained from butter lecithin. it was found that the results conformed in all respects to the deseriptions of this periodide. The accompanying plate of photomierographs (Plate VI) shows the characteristic crystals and oily droplets of the periodide formed by the choline from butter. In addition to this test it was shown that the small amount of choline obtained woukl give off a slight but distinct fishy odor on being heated with solid caustic soda.

\section{AMOUNT' OF LHOITIIN IN BITTIEIR}

Sine the qualitative tests seemed to leave no room for doubt as to the presence of lecithin in butter, the next step was the quantitative estimation of this substanee. In view of the evident diffieulty in securing an absolutely pure lecithin free from other phosphatides, the estimations were based on the phosphorus content of extracts and the amount of lecithin calculated according to the formula of the distearyl type. The results of such determinations on various types of butter made from different lots of eream are showi in table 2 :

TABLE 2. Lacithin Content in Various Butters*

\begin{tabular}{|c|c|c|c|c|}
\hline Sample & $\begin{array}{l}\text { Type of cream from } \\
\text { which butter was made }\end{array}$ & $\begin{array}{l}\text { Age of } \\
\text { butter } \\
\text { (days) }\end{array}$ & $\begin{array}{c}\mathrm{P}_{2} \mathrm{O}_{5} \\
\text { (per cent) }\end{array}$ & $\begin{array}{l}\text { Lecithin, } \\
\text { distearyl } \\
\text { type } \\
\text { (per cent) }\end{array}$ \\
\hline $\begin{array}{l}1 \ldots \ldots \ldots \\
2 \ldots \ldots \ldots \\
3 \ldots \ldots \ldots \\
4 \ldots \ldots \ldots \ldots\end{array}$ & $\begin{array}{l}\text { Raw sweet . . . . . . . } \\
\text { Pasteurized sweet . . . } \\
\text { Raw ripened. . . . . . } \\
\text { Pasteurized ripened. . }\end{array}$ & $\begin{array}{l}6 \\
6 \\
6 \\
6\end{array}$ & $\begin{array}{l}0.0127 \\
0.0127 \\
0.0122 \\
0.0075\end{array}$ & $\begin{array}{l}0.0723 \\
0.0723 \\
0.06933 \\
0.0433\end{array}$ \\
\hline $\begin{array}{l}5 \ldots \ldots \\
6 \ldots \ldots \\
7 \ldots \ldots \ldots\end{array}$ & $\begin{array}{l}\text { Raw sweet. .......... } \\
\text { Pasteurized sweet . . . . } \\
\text { Raw ripened. . . . . } \\
\text { Pasteurized ripened. . }\end{array}$ & $\begin{array}{l}48 \\
48 \\
48 \\
18\end{array}$ & $\begin{array}{l}0.0092 \\
0.0120 \\
0.0056 \\
0.0053\end{array}$ & $\begin{array}{l}0.0 \pi 22.2 \\
0.06822 \\
0.0154 \\
0.0171\end{array}$ \\
\hline $\begin{array}{l}9 \ldots \ldots \\
10 \ldots \ldots \\
11 \ldots \ldots \ldots \\
12 \ldots \ldots\end{array}$ & $\begin{array}{l}\text { Raw sweet .......... } \\
\text { Pasteurized sweet. . . . . } \\
\text { Raw ripened. ........ } \\
\text { Pasteurized ripened .... }\end{array}$ & $\begin{array}{l}72 \\
72 \\
72 \\
72\end{array}$ & $\begin{array}{l}0.0111 \\
0.00 \mathrm{~s} !) \\
0.0083 \\
0.0095\end{array}$ & $\begin{array}{l}0.063 i 3 \\
0.050 \% \\
0.0471 \\
0.05+1)\end{array}$ \\
\hline
\end{tabular}

* These figures were furnished by J. T. Cusick, chemist for the state Department of Agriculture, located at Cornell University. 

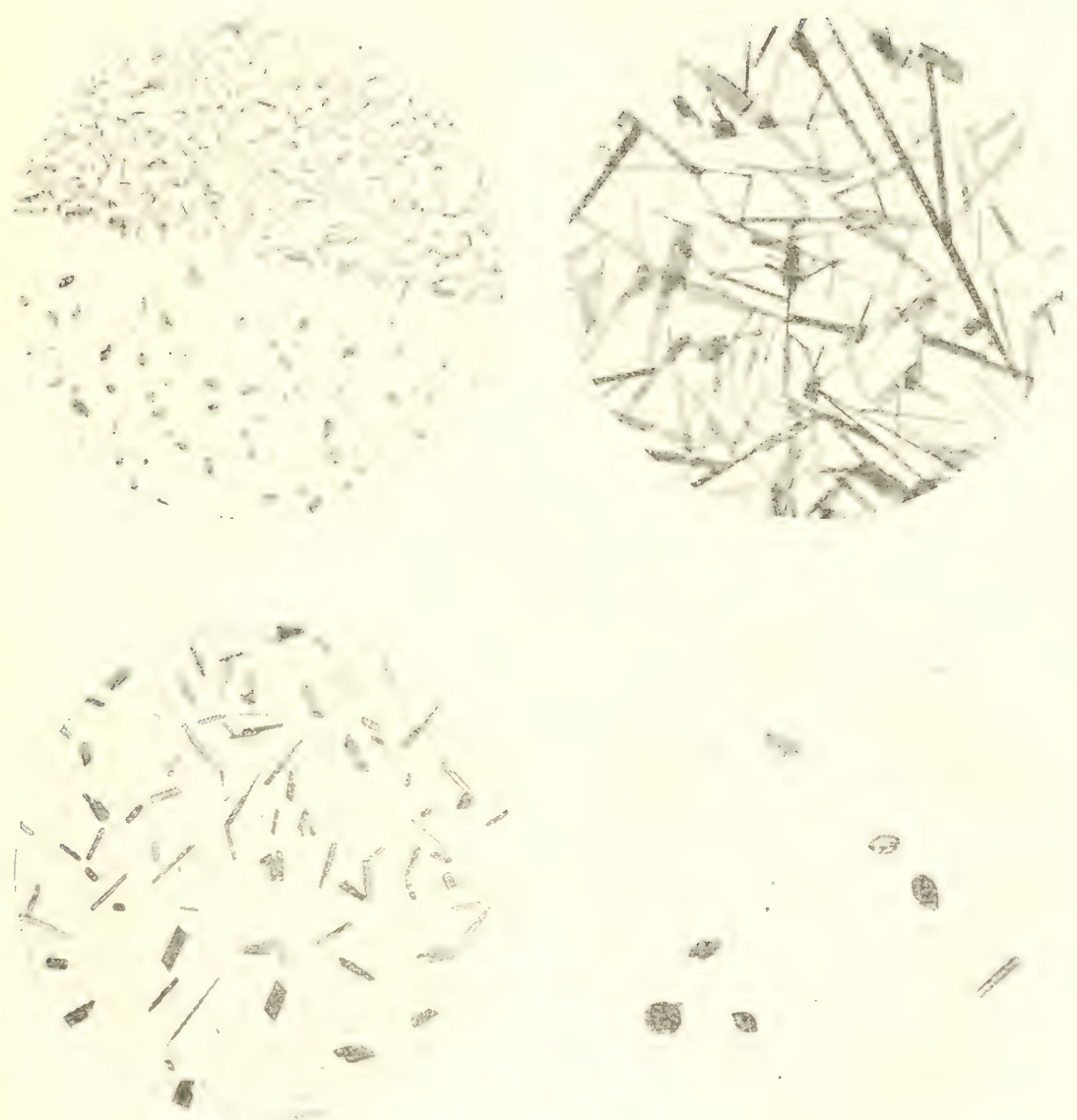

CHOLINE PER:ODIDE (AYTTALS OBTAINED FLOM CHOLTNE OF BUTTER LECITHN I'hotonicrompaplss, $\times 3 \mathrm{so}$ 

A study of table 2 show's a fairly constant lecithin content in butter from various lots of eream and in rlifferent types of butter made from the same lot of eream. There is one feature, howerer, which is worthy of note, and this is that in most instances there is a tendeney toward a lower lecithin content in the ripened-eram butter than in that made from umipened cream. This may be significant in the light of the statement by Hammarsten and Hedin (1915), that lecithin is deromposed by dilute acids and enzymes. This fact applied to these results might indieate that the acidity of the cream slowly decomposed the leceithin, and its decomposition products, particularly the alycerophosphorie acid, were washed out with the butermilk. If such were the anse it would be rery easy to account for the lower phosiphorus content in sour-eream butter.

\section{TRIMFTHYLAMIN SAMTA OF 'THE FATY A CIIDS}

It was decided that before an attempt wats made to correlate trimethylamine with the fishy flavor of butter, this substanee should be prepared in a pure state and those chatacteristics detemined which might have a bearing on this particalar problem. Trimethylumine was made by heating 50 grams of ammonium chloriste and 4.40 grams of a $4(0-p)$ er-ecent

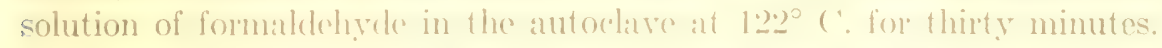
Any exeess formaldehyede was then expedled and the trimethylanime liberated from its hydrochloride by distilling from an alkaline solution. A 10 -per-eent solution was casily obtained at the ordinary temperature and pressure. The trimethylamine thus produred was combined with lactic, butyrie, oleice, and stearie acids, and also with the mixed soluble and insoluble fatty acids obtained from butter aceording to the proesdure outlined by Browne (1899). While the properties of these ated addition products seemed to offer an interesting field for study, only such of their characteristics are reseorded here as mieht have a direcet relationship to the fishy flavor in buter, namely, their stability, volatility, and behavior in the presenee of sodium ehloriele.

The lactic-acid combination with trimethylamine proved to be a relatively stable oily liepuid possesing no chameteristic taste other than that shown by many common salts. The odor, esperially after the licquid had been standing in a stoppered bottle, seemed to be slightly fishy. Evidence on this point is not eonchusive beratse it is possible that this was due to exeess trimethylumine adcled at the time of neutralization and 
not shown by the indieator used. Ineating secmed to intensify the odor to some extent, which would indicate instability at high temperatures.

The butyrie-acid combination with trincthytamine was a substance extremely volatile at ordinaty tomperatures. The ordor greatly resembled fish oil; the faste resembling this product was manifest only when very small amounts were used. and then not regularly.

Oleic acid and trimethylanine formod a soft soap which was very unstable as evideneed by the liberation of the trimethytamine; the greater the dilution, howerer, the lese this condition wats manifested. This soap could not he shtained entirely free from water, even at the ordinary temperatures, berause of the simultanerous giving-off of trimethylamine and water. This lithration was sum that nothing remained but the free acid. Furthermore, on the addition of soclimm ahlorde to its water solution, the sodium iom readily replaced the trincthylamine radical, with the consecquent precipitation of the sodium soalp and the formation of trimethylamine hydrochloride.

The trimethrlanine stantate showerl the same aharacteristies as the oleie soap, but to an cren gleater extent. Trincthylamine was constantly given off in latre guantiter, and the only way in which it conld be handled ats a seap) was in a mixture of alcohol and water in a tightiy stoppered bottle.

The mixed soluble and insolulle fatty acids combined with trimethylamine showed the same general characteristice as the butyric and stearie combinations, respectively.

The instability of the conblinations of fatty actel and trimethylamine can undoubtally be aceomented for hy the taxed that they are addition products in which the trivalent nitrogen of the latter substance changes to the pentavatent condition in the puresenee of an aleid. 'The fact that these are weak ariels with relulively latree molecules is probably also significant. It was oblecerved that the hyedrochloride was more stable than the above salts, and that the sulfate wats even more stable than the hydrechloride. This instability of the fatty-aced eombimations and their reatetion in the presence of soclimm ehlorite may have an important bearing on the relation of trinethylanine to fishiness in butter, and may be of particular signifienese in explaining why the flavor is usually found in salted butter. As yet, however, the relationship is not. clear. 


\section{EFEECT OF WOIKJNG TRIALTHYLAMTYE SILTS OF TIL FATTY}

A(II) INTO BLTIER

The charactor of the frimethylamine salts of the fatty acids in pure state scemed to justify the following serves of experinents, in which these salts are incorporaterl into varions types of hatcer for the purpose of determining the possibility of their prochume the fishy flavor in the presence of hutterfit. In vieny of the decirability of ineorporating the trimethylamine in logical amounts, the following plan was adopted:

The latgest quantity of fecithin foportert in row's milk by Nerking and Haensel (1908) was used as the bavis of calculation. These authors report 0.116 per cont as the largest amount found in seventeen samples. For the calculations of this experinemi, this wats assumed to be lecithin of the distearyl type, and it was lunther atsumed to be pure lecithin with the empirical formula asigned to the tyge named. (iranting these assumptions, this amount would yide on complete decomposition the equivalent of $8 \tilde{5}$ jarts jere nitlion of trimethylamine. This substance alone on in acid combination was therefore addort to cream, wash water, or butter on this baxis. It is rerpericient that berause of the volatility of some of the materials and leesatse of meehanical loss, none of the samples of butter when ampleterl would eontain 8.5 parts pere million of trimethytanine. The method of arriving at the quantity to be added

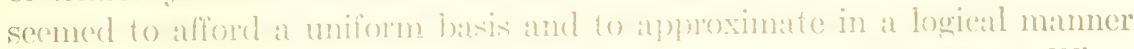
the amount of this substanee that might be produesd in butter. When the fatty acids were weed abone they were adder in cquantities equivalent to the amounts adder! in the corresponding trinethylannine sults. The addition of the acdets was merely for the pmovece of ehecking against the trimethylimine.

In tables 3 to 9 indusive are shown the comments of various judges on different types of hutfere containing trimethylamine added as already indieated and incorpolated hy various means. Because of the great importanee of the personal faretor in juelging hutter, an effort was made

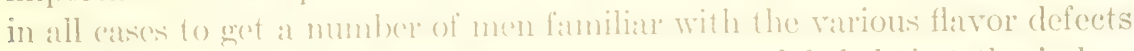
of the protuet. In all cases the samples werese labeled that the judges had no knowlerfere of their eontents. They were instructed to comment on the flavor and to work inderendently of one another, and it is believed that this injunction was carricel ont. In presenting the results in tabulax form the auther has intentionally onited comments having no direct bearing on the fishy flavol. 
TABLF, 3. FfFect ox the Flayor of Butter, of Adding 'Timethylamine and Fatty

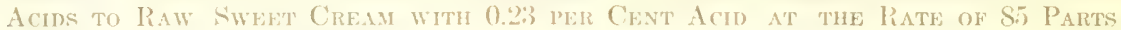
PER MILIION OF THE FoIRMER

( $S$ ' indicates salted butter)

\begin{tabular}{|c|c|c|c|c|c|c|c|}
\hline \multirow{2}{*}{ Sample. } & \multirow{2}{*}{ Miterial ardded } & \multicolumn{6}{|c|}{ Comments by judges } \\
\hline & & No. 1 & $N_{0} . " 2$ & No. 3 & No. 4 & No. 5 & No. 6 \\
\hline $\begin{array}{l}A S \\
A\end{array}$ & Nothing . . . . . . . . & & (lils & & ()ily & $\begin{array}{l}\text { Oily } \\
\text { Wili }\end{array}$ & \\
\hline $\begin{array}{l}1 \mathrm{AS} \\
1 \mathrm{~A}\end{array}$ & $\begin{array}{l}\text { Trimethylamine....... } \\
\text { Trinethylamine...... }\end{array}$ & $\begin{array}{l}\text { Oily } \\
\text { Fishy }\end{array}$ & Fishy: & & Fishy & & Fïshy \\
\hline $\begin{array}{l}2 A S \\
2 A\end{array}$ & $\begin{array}{l}\text { Trimethylamine lacetate . . . . . } \\
\text { Trimethylamine liketate . . . . }\end{array}$ & Fishy & ()ily & Fishy & frishyl & & \\
\hline $\begin{array}{l}3 \mathrm{AS} \\
3 \mathrm{~A}\end{array}$ & $\begin{array}{l}\text { Trimethylamine butyrate...... } \\
\text { 'Trimethylanine butyrate. . . }\end{array}$ & $\begin{array}{l}\text { Fishy } \\
\text { Fishy }\end{array}$ & $\begin{array}{l}\text { Fishy: } \\
\text { ()ily }\end{array}$ & Fishy & $\mid$ issloy & & \\
\hline $\begin{array}{l}4 A \\
4 A\end{array}$ & $\begin{array}{l}\text { Trimethylamine oleate ....... } \\
\text { Trimethylanine oleate . . . . . }\end{array}$ & rishy & & & lishyl| & & \\
\hline $51=$ & $\begin{array}{l}\text { Trimethylamine stearate. } \\
\text { Trime hivamenes wh at }\end{array}$ & $\begin{array}{l}\text { lishy } \\
\text { l wat }\end{array}$ & & & & & \\
\hline $6 \mathrm{AS}$ & $\begin{array}{l}\text { Trime thylamine and soluble fatty } \\
\text { acrids }\end{array}$ & & & Finloy| & Fislyy & & Fishy \\
\hline 6.1 & $\begin{array}{l}\text { Trimethylamme and solubis fitt? } \\
\text { in-i.i. }\end{array}$ & $|i-1|\rangle$ & $1 \mathrm{i} \cdot 1 \mathrm{~s}$ & & & & \\
\hline $7 \mathrm{AS}$ & 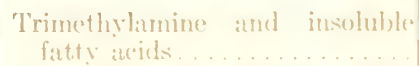 & & lokhy & Fishyl & liis] & & Fishy \\
\hline 71 & $\begin{array}{l}\text { Trimethylamine and insoluble } \\
\text { fatty acids................. }\end{array}$ & & (Oily & & & & \\
\hline $31=$ & 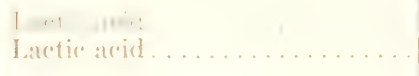 & Fishy & ()ily & & $1-1$ & & \\
\hline $\begin{array}{lll}3 \mathrm{~A} \\
4 \mathrm{~A}\end{array}$ & $\begin{array}{l}\text { Butyric acid . . . . . . . } \\
\text { Butyric acid . . . . . }\end{array}$ & $\begin{array}{l}\text { Fishy } \\
\text { lishy }\end{array}$ & & & ()ily & & \\
\hline 11) A & 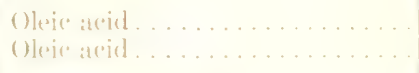 & & Fishy & Fistly & lrishy & & \\
\hline $11 \AA$ & $\begin{array}{l}\text { Solublue fatty acids . . . . . } \\
\text { soluble fattio aceds. . . . }\end{array}$ & & & & & & \\
\hline $\begin{array}{l}12 \mathrm{~A} \\
121\end{array}$ & 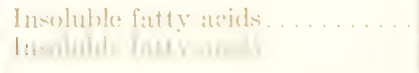 & & & & & & \\
\hline
\end{tabular}


TABLE 4. EfFect on the Flavor of Butter, of Adding Trimethylamine and Fatpy Acids to Raw Sweet Cieam with 0.23 per Cext Acid at the Rate of 85 Parts per Million and Then Washing the Butter in Water Containing the Same Concentration of the Various Substances

( $S$ indicates salted butter)

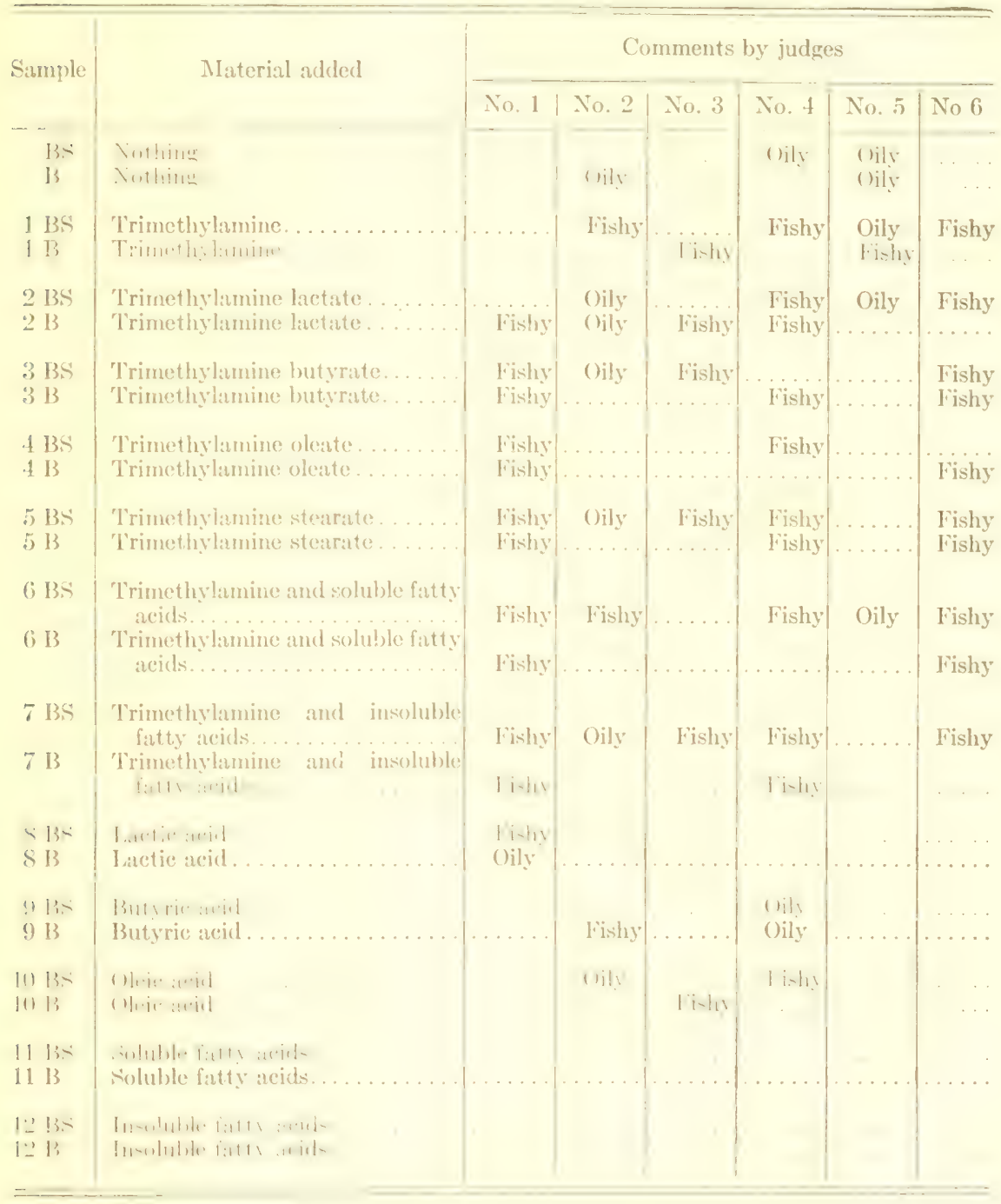




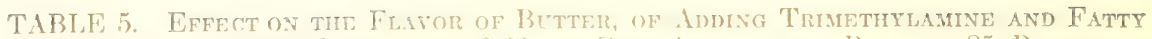

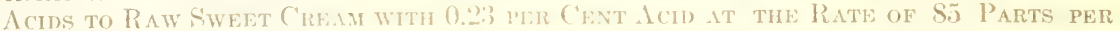
Mildion and Then Working the sumstayces Directly into the Butrer at the SAME RATF

(h indieates saited butter)

\begin{tabular}{|c|c|c|c|c|c|c|c|}
\hline \multirow{2}{*}{ Sample } & \multirow{2}{*}{ Material ankled } & \multicolumn{6}{|c|}{ C'omments by judges } \\
\hline & & Xo. 1 & No. 2 & No. 3 & No. 4 & No. 5 & No. 6 \\
\hline $\mathrm{Cr}$ & Jothing . . . . . . . & & oii & & ()ily & $\begin{array}{l}\text { Oily } \\
\text { Oily }\end{array}$ & \\
\hline $\begin{array}{l}1 \mathrm{CA} \\
1 \mathrm{C}\end{array}$ & $\begin{array}{l}\text { Trimethylamine. . . . . . . . } \\
\text { Trimethylumine. . . . . . . }\end{array}$ & I ishy & $\begin{array}{l}\text { Fishy } \\
\text { l"ishy }\end{array}$ & lïhligy & $\begin{array}{l}\text { Fishy } \\
\text { Fishy }\end{array}$ & $\begin{array}{l}\text { lishy } \\
\text { Hishy }\end{array}$ & $\begin{array}{l}\text { Fishy } \\
\text { Iishy }\end{array}$ \\
\hline $\begin{array}{l}2 \mathrm{CN} \\
2 \mathrm{C}\end{array}$ & $\begin{array}{l}\text { Trimethylanime liutate . . . . . } \\
\text { Trimethylanine lavetate. . . . }\end{array}$ & 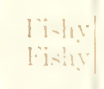 & $\begin{array}{l}\text { Jishy } \\
\text { Fishy }\end{array}$ & $\begin{array}{l}\text { Fishy } \\
\text { lishy }\end{array}$ & Wishy & $\begin{array}{l}\text { Tishy } \\
\text { Fishy }\end{array}$ & $\begin{array}{l}\text { Fishy } \\
\text { ivishy }\end{array}$ \\
\hline $\begin{array}{l}3 \mathrm{Cr} \\
3 \mathrm{C}\end{array}$ & $\begin{array}{l}\text { Trimethylanine hutyrate. . . } \\
\text { Trimethylanine butyrate. . }\end{array}$ & $\begin{array}{l}\text { Winhy } \\
\text { lishly }\end{array}$ & $\begin{array}{l}\text { Tis ly } \\
\text { rishy }\end{array}$ & $\begin{array}{l}\text { lishyy } \\
\text { lisishy }\end{array}$ & rishy & Iishy & $\begin{array}{l}\text { Fishy } \\
\text { Fishy }\end{array}$ \\
\hline $\begin{array}{l}4 \mathrm{CK} \\
4 \mathrm{C}\end{array}$ & $\begin{array}{l}\text { Trimethytamine wieate . . . . . . } \\
\text { Trimethylamine oleate. . . . . }\end{array}$ & Why & Hishy & $\begin{array}{l}\text { lishy } \\
\text { lishy }\end{array}$ & $\begin{array}{l}\text { lishy } \\
\text { lishy }\end{array}$ & & \\
\hline $\begin{array}{l}5 \mathrm{Cs} \\
5 \mathrm{C}\end{array}$ & $\begin{array}{l}\text { Trimethylamine stearate . . . . } \\
\text { Trimethylamine stearate . . . }\end{array}$ & $\begin{array}{l}\text { lisklyy } \\
\text { I.isly }\end{array}$ & & & $\begin{array}{l}\text { Fishy } \\
\text { Wishy }\end{array}$ & $\begin{array}{l}\text { Fishy } \\
\text { W }\end{array}$ & $\begin{array}{l}\text { Fishy } \\
\text { Fishy }\end{array}$ \\
\hline (i) $\mathrm{CS}$ & Trimethylamine and solul, le fatty & 11170 & & $1 i-11:$ & & $\mid i-h y$ & \\
\hline $6 \mathrm{C}$ & $\begin{array}{l}\text { Trimethylamine and soluble fut ty } \\
\text { st. }\end{array}$ & InIIV & & $|i-| 1 \mid$ & & lixhy & \\
\hline $7 \mathrm{Cs}$ & 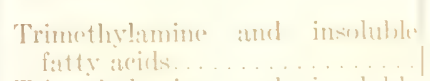 & lishy & & Fishy & Fishy & Fishy & Fishy \\
\hline $7 \mathrm{C}$ & $\begin{array}{l}\text { Trimethylamine and in-olubla } \\
\text { fattly acids............... }\end{array}$ & lishyl & & & & Fishy & Fishy \\
\hline $\begin{array}{l}\& C \\
\& C\end{array}$ & 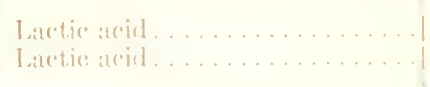 & ()ily & & Fishy & & & $\begin{array}{ll}-\cdots \\
\cdots \cdots\end{array}$ \\
\hline $\begin{array}{l}9 \mathrm{CS} \\
9 \mathrm{C}\end{array}$ & $\begin{array}{l}\text { Butyrie acid . . . . . . . } \\
\text { Butyric acid . . . . . }\end{array}$ & $\begin{array}{l}\text { Fishy } \\
\text { Finity }\end{array}$ & lishy & & Fishy & & \\
\hline $\begin{array}{l}\text { 10) }(x) \\
\text { liic }\end{array}$ & () Here reid. & & & $\begin{array}{l}\text { Wishy| } \\
\text { | i-li! }\end{array}$ & $\begin{array}{l}\text { Fishy } \\
\text { li-h!! }\end{array}$ & & \\
\hline $\begin{array}{l}11(\vdots \\
11(\vdots\end{array}$ & $\begin{array}{l}\text { suduble fatty acide...... } \\
\text { robublo fatty acids..... }\end{array}$ & & & & & & \\
\hline 120 & 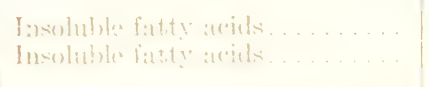 & $\begin{array}{l}\text { ()ily } \\
\text { ()ily }\end{array}$ & & & & (Oily & \\
\hline
\end{tabular}


TABle 6. EfFect on the Flavor of Butter Mlade hrom Pasteurized Ripened Cream with 0.32 per Cent Acid, Working Tizmethylamine and Fatty Acids Directly into the Butrer it the R.ite of 8j Patrts per Million

$$
\text { (s) indicates salted buiter) }
$$

\begin{tabular}{|c|c|c|c|c|c|c|c|}
\hline \multirow{2}{*}{ Sample } & \multirow{2}{*}{ Mizterial added } & \multicolumn{6}{|c|}{ Comments by judges } \\
\hline & & No. 1 & $N_{0}, 2$ & No. 3 & No. & No. $5 \mid 1$ & No. 6 \\
\hline 1) & $\begin{array}{l}\text { Sinthinge } \\
\text { Sinthing }\end{array}$ & & & & & & \\
\hline $\begin{array}{ll}11) \\
11)\end{array}$ & $\begin{array}{l}\text { Trimeth! lamis.. } \\
\text { Trimethylatus.. }\end{array}$ & & Mishy & $\begin{array}{l}\text { Fishy } \\
\text { Hishy }\end{array}$ & $\begin{array}{l}\text { Fishy } \\
\text { lishy }\end{array}$ & $\begin{array}{l}\text { Fishy. } \\
\text { Fishy. }\end{array}$ & \\
\hline $\begin{array}{l}2 \mathrm{D} \\
2 \mathrm{D}\end{array}$ & 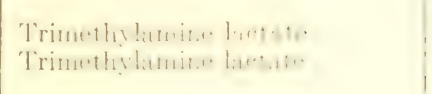 & & & & & & \\
\hline $\begin{array}{l}3 \mathrm{DL} \\
3 \mathrm{D}\end{array}$ & $\begin{array}{l}\text { Trimethylamine butyrate...... } \\
\text { Trimethylamine butyrate..... }\end{array}$ & $\begin{array}{l}\text { Oily } \\
\ldots\end{array}$ & Irishy & & $\begin{array}{l}\text { lill } \\
\text { l iill }\end{array}$ & $\begin{array}{l}\mid \text { Iinlyy } \\
|\mathrm{i}-\mathrm{i}, \mathrm{y}|\end{array}$ & $\begin{array}{l}\text { Finhy } \\
\text { Hat }\end{array}$ \\
\hline $\begin{array}{l}4 \mathrm{D} \\
4 \mathrm{D}\end{array}$ & 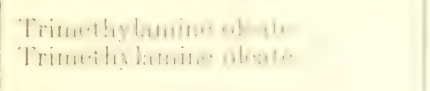 & & & & & & \\
\hline $\begin{array}{l}5 \mathrm{D} \\
5 \mathrm{D}\end{array}$ & $\begin{array}{l}\text { Trimethylamine ste urate. . . . } \\
\text { Trimethylanine stearate. . . }\end{array}$ & init & & & $\begin{array}{l}\text { 1:1:y } \\
\text { Wil: }\end{array}$ & 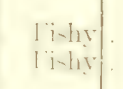 & \\
\hline $6 \mathrm{Ds}$ & $\begin{array}{l}\text { Trimethylamine and soluble fatty } \\
\text { acid. }\end{array}$ & & & lility & & & \\
\hline $6 \mathrm{D}$ & 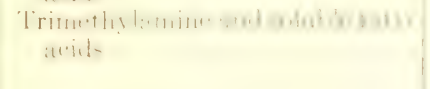 & & & & & & \\
\hline $711:$ & $\begin{array}{l}\text { Trimethylamine and insoluble } \\
\text { fatty acids............... }\end{array}$ & & $|n i|$, & & & & \\
\hline 711 & $\begin{array}{l}\text { Trimethylamine and incoluble } \\
\text { fatt an } 4\end{array}$ & & linily & & & & \\
\hline $\begin{array}{l}8112 \\
811\end{array}$ & 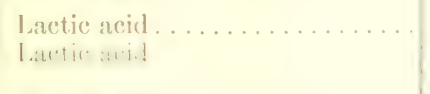 & $(\mathrm{Bl})$ & 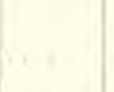 & & & & \\
\hline $\begin{array}{ll}41 M \\
1 & 11\end{array}$ & $\begin{array}{l}\text { Buty rie and } \\
\text { Butyrie atil }\end{array}$ & l inisy & $\begin{array}{l}\text { I why } \\
\text { I'isluy }\end{array}$ & & & & \\
\hline $\begin{array}{l}\text { (1) 1): } \\
\text { (1) 1) }\end{array}$ & 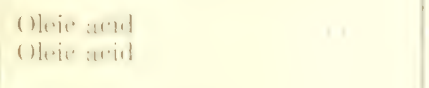 & & & $\begin{array}{l}\text { lishyl } \\
\text { lith }\end{array}$ & & & \\
\hline $\begin{array}{ll}11 & 11 \\
11 & 11\end{array}$ & $\begin{array}{l}\text { Soluble fatty acids.......... } \\
\text { soluble finty acids........ }\end{array}$ & & & & & & \\
\hline $\begin{array}{l}1: 112 \\
1211\end{array}$ & $\begin{array}{l}\text { Insoluble fatty acids. } \\
\text { Insoluble fatty acids }\end{array}$ & $\begin{array}{l}\text { ()ily. } \\
\text { ()ily }\end{array}$ & & & & $\begin{array}{l}\text { rily } \\
\text { rily }\end{array}$ & \\
\hline
\end{tabular}


Tabie 7. Effect on the Flavor of Butter Made from Pasteurized Sweet Cream with 0.16 per Cent Acid, Working Trimethylamine and Fatty Acids Directly into the Butter at the R.ate of 85 Paits per Million

( $S$ indicates salted butter)

\begin{tabular}{|c|c|c|c|c|c|c|c|}
\hline \multirow{2}{*}{ Sample } & \multirow{2}{*}{ Material adde: } & \multicolumn{6}{|c|}{ Comments by judges } \\
\hline & & No. 1 & No. 2 & No. 3 & No. 4 & No. 5 & No. 6 \\
\hline $\begin{array}{l}\mathrm{ES} \\
\mathrm{E}\end{array}$ & $\begin{array}{l}\text { Nothing. . } \\
\text { Nothing.. }\end{array}$ & & & & & & \\
\hline $\begin{array}{l}1 \mathrm{ES} \\
1 \mathrm{E}\end{array}$ & $\begin{array}{l}\text { Trimethylamine . . . . . . . . . } \\
\text { Trimethylamine......... }\end{array}$ & $\begin{array}{l}\text { Fishy } \\
\text { Fishy }\end{array}$ & $\begin{array}{l}\text { Fishy } \\
\text { Fishy }\end{array}$ & Fishy & Fishy & & $\begin{array}{l}\text { Fishy } \\
\text { Fishy }\end{array}$ \\
\hline $\begin{array}{l}2 \mathrm{ES} \\
2 \mathrm{E}\end{array}$ & $\begin{array}{l}\text { Trimethylamine lactate. . . . . . } \\
\text { Trimethylamine lactate. . . . }\end{array}$ & Fishy & $\begin{array}{l}\text { Fishy } \\
\text { Fishy }\end{array}$ & Fishy & Fishy & & $\begin{array}{l}\text { Fïhy } \\
\text { Fishy }\end{array}$ \\
\hline $\begin{array}{l}3 \mathrm{ES} \\
3 \mathrm{E}\end{array}$ & $\begin{array}{l}\text { Trimethylamine butyrate...... } \\
\text { Trimethylamine butyrate.... }\end{array}$ & Fishy & $\begin{array}{l}\text { Fishy } \\
\text { Fishly }\end{array}$ & Fishy & Fishy & Fishy & $\begin{array}{l}\text { Fishy } \\
\text { Fishy }\end{array}$ \\
\hline $\begin{array}{l}4 \mathrm{ES} \\
4 \mathrm{E}\end{array}$ & $\begin{array}{l}\text { 'Trimethylamine oleate . . . . . . . } \\
\text { Trimethylamine oleate . . . . . }\end{array}$ & Fishy & $\begin{array}{l}\text { Fishy } \\
\text { Fishy }\end{array}$ & & & & lishy \\
\hline $\begin{array}{l}5 \mathrm{ES} \\
5 \mathrm{E}\end{array}$ & $\begin{array}{l}\text { Trimethylamine stearate. } \\
\text { Trimethylamine stearate. }\end{array}$ & & $\begin{array}{l}\text { Fishy } \\
\text { ()ily }\end{array}$ & Fishy & $\begin{array}{l}\text { Fishy } \\
\text { Fishy }\end{array}$ & Fishy & $\begin{array}{l}\text { Fishy } \\
\text { Tishy }\end{array}$ \\
\hline $6 \mathrm{Es}$ & $\begin{array}{l}\text { Trime thy lamine and soluble fatty } \\
\text { icids. }\end{array}$ & Fishy & & & Fishyl & Fijshy & \\
\hline $6 \mathrm{E}$ & $\begin{array}{l}\text { Trimethylimine and soluble fatty } \\
\text { itcids.................. }\end{array}$ & & Fishy & Wishy & Fishy| & 175ry & tosts \\
\hline $7 \mathrm{ES}$ & $\begin{array}{l}\text { Trimethylamine and insoluble } \\
\text { tat1s }\end{array}$ & $|i=| 11$ & $|i-h|$ & & & low & \\
\hline $7 \mathrm{E}$ & $\begin{array}{l}\text { T'rimethylamine and insoluble } \\
\text { falt? }\end{array}$ & & $1 i-1$ ! & & & & \\
\hline $\begin{array}{l}S 1:- \\
8 \mathrm{E}\end{array}$ & $\begin{array}{l}\text { Lan th acid } \\
\text { Latetie acid . . . . . . . }\end{array}$ & & $\begin{array}{l}\text { li i-h! } \\
\text { lishy| }\end{array}$ & & & & \\
\hline $\begin{array}{l}9 \mathrm{EK} \\
9 \mathrm{E}\end{array}$ & $\begin{array}{l}\text { Butyrie acid . . . . . . . } \\
\text { Butyric acid ........ }\end{array}$ & $\begin{array}{l}\text { Fishy } \\
\text { Fishy }\end{array}$ & & $\begin{array}{l}\text { Fishy } \\
\text { Fishy }\end{array}$ & Fishy & Fishy & Fishy \\
\hline $\begin{array}{l}10 \mathrm{ES} \\
10 \mathrm{HS}\end{array}$ & $\begin{array}{l}\text { Oleic acid . . } \\
\text { Oleic acid.. }\end{array}$ & & & & $\begin{array}{l}\text { Fishy } \\
\text { Fishy }\end{array}$ & & \\
\hline $\begin{array}{l}11 \mathrm{ES} \\
11 \mathrm{E}\end{array}$ & $\begin{array}{l}\text { Soluble fatty acids........ } \\
\text { soluble fatty acids....... }\end{array}$ & & & & l'ishy & Fishy & \\
\hline $\begin{array}{l}12 \mathrm{ES} \\
12 \mathrm{E}\end{array}$ & $\begin{array}{l}\text { Insoluble fatty acids ...... } \\
\text { Insoluble fatty acids..... }\end{array}$ & $\begin{array}{l}\text { Oily } \\
\text { Oily }\end{array}$ & & & & & Oily \\
\hline
\end{tabular}


Tabie 8. Effect on the Flator of Butter Made from Pasteurizen Ripened Cream with 0.38 per Cent Acid, Working Trinethylamine and Fatty Acids Directhy into the Butter at the Rate of 40 Patts per Million

( $S$ indicates salted butter)

\begin{tabular}{|c|c|c|c|c|c|}
\hline \multirow{2}{*}{ Sample } & \multirow{2}{*}{ Material added } & \multicolumn{4}{|c|}{ Comments by judges } \\
\hline & & No. 1 & No. 2 | & No. 3 & No. 4 \\
\hline $\begin{array}{l}\text { FS } \\
\mathrm{I}\end{array}$ & $\begin{array}{l}\text { Nothing. } \\
\text { Vinthing }\end{array}$ & $\ldots \ldots$ & & & \\
\hline $\begin{array}{l}1 \mathrm{FS} \\
1 \mathrm{~F}\end{array}$ & $\begin{array}{l}\text { Trimethylamine } \ldots \ldots \ldots \ldots \ldots \ldots \ldots \ldots \ldots \ldots \ldots \ldots \\
\text { Trimethylamine } \ldots \ldots \ldots \ldots \ldots \ldots \ldots\end{array}$ & $\begin{array}{l}\text { Oily } \\
\text { Oily }\end{array}$ & $\begin{array}{l}\text { Oily } \\
\text { Fishy }\end{array}$ & $\begin{array}{l}\text { Fishy } \\
\text { Oily }\end{array}$ & \\
\hline $\begin{array}{l}2 \mathrm{FS} \\
2 \mathrm{~F}\end{array}$ & 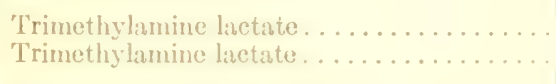 & $\begin{array}{l}\text { Fishy } \\
\text {. }\end{array}$ & Fishy & $\begin{array}{l}\text { Fishy } \\
\cdots\end{array}$ & \\
\hline $\begin{array}{l}3 \mathrm{FS} \\
3 \mathrm{~F}\end{array}$ & 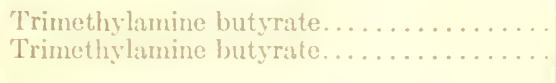 & $\begin{array}{l}\text { Fishy } \\
\text { W. }\end{array}$ & $\begin{array}{l}\text { Fishy } \\
\text { rishy }\end{array}$ & $\begin{array}{l}\text { Fishy } \\
\text { Fishy }\end{array}$ & $\begin{array}{l}\text { Fishy } \\
\text { Fishy }\end{array}$ \\
\hline $\begin{array}{l}4 \mathrm{FS} \\
4 \mathrm{IT}^{\mathrm{T}}\end{array}$ & $\begin{array}{l}\text { Trimethylamine oleate } \ldots \ldots \ldots \ldots \ldots \ldots \\
\text { Trimethylamine oleate } . \ldots \ldots \ldots \ldots \ldots\end{array}$ & Oily & & & ... \\
\hline $\begin{array}{ll}i & 1 \\
i & 1\end{array}$ & 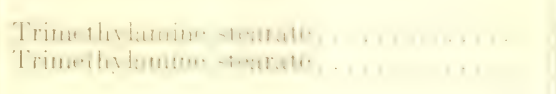 & & & & \\
\hline $6 \mathrm{FS}$ & Trimethylamine and soluble fatty acids. . & Oily & & Fishy & Fishy \\
\hline $\begin{array}{l}7 \mathrm{Fs} \\
7 \mathrm{~F}\end{array}$ & $\begin{array}{l}\text { Trimethylamine and insoluble fatty acids... } \\
\text { Trimethylamine and insoluble fatty acids.. }\end{array}$ & & & & $\ldots \ldots$ \\
\hline $81:$ & $\begin{array}{l}\text { Lawi a ini } \\
\text { Lactic acid . . . . . . . . . }\end{array}$ & & & & toriy \\
\hline $\begin{array}{l}111 \% \\
9 \mathrm{~F}\end{array}$ & $\begin{array}{l}\text { Bust rie ard } \ldots \\
\text { Butyric acid } \ldots \ldots \ldots \ldots \ldots \ldots \ldots\end{array}$ & & & & \\
\hline $\begin{array}{l}\text { 11) } \mathrm{li} \\
10 \mathrm{~F}\end{array}$ & $\begin{array}{l}\text { (1) ir and } \\
\text { Oleic acid. }\end{array}$ & & & & \\
\hline
\end{tabular}




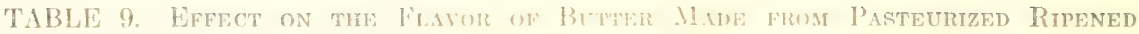

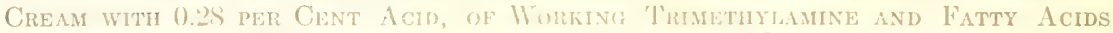

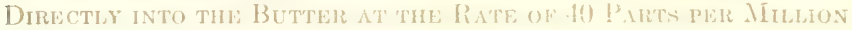

(s' indieates stille+l butter)

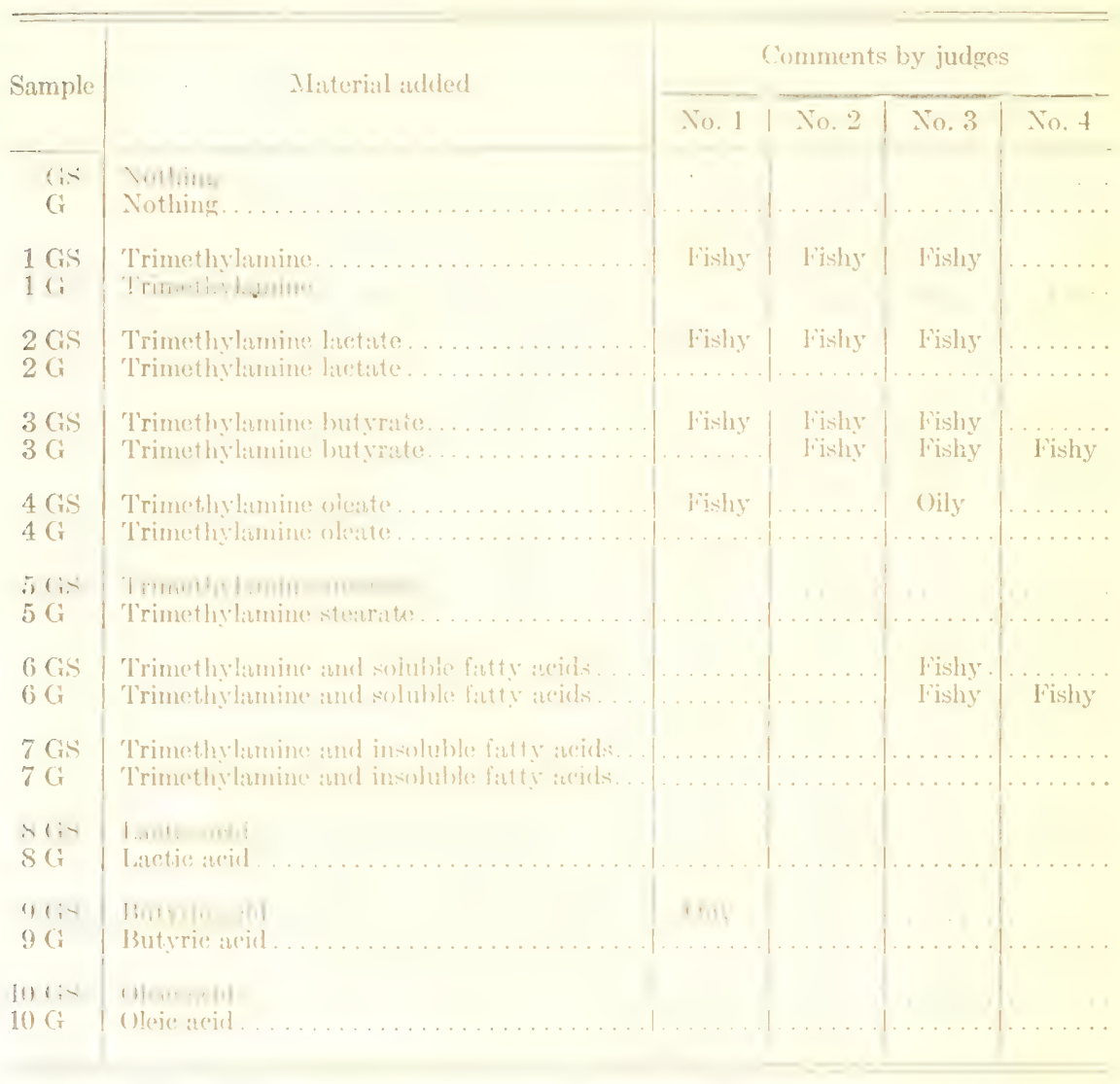

All of the simples of butter renperentod in tables :3 to 9 inchusive were seored from three to fire disy after making. They were then placed in storage for different lengthe of time and resecored by two or more judges. The resilts of this examination are shown in table 10. In this table are listerl only those sinnples showing a fishy flavor by unanimous opinion of the persons jutging them. 
TABLE 10. Presence of Fishy Flivot after Stordge, in Butter to Which 'Trimethylame Had Been Added at the 'Time of Makrng

\begin{tabular}{|c|c|c|c|c|}
\hline \multirow{2}{*}{ Sample } & \multirow{2}{*}{ Material added } & \multirow{2}{*}{$\begin{array}{l}\text { Age } \\
\text { (days) }\end{array}$} & \multicolumn{2}{|c|}{ Comments by judges } \\
\hline & & & $\begin{array}{l}\text { At time of } \\
\text { making }\end{array}$ & After storage \\
\hline $\begin{array}{l}3 \mathrm{CS} \\
3 \mathrm{DS} \\
3 \mathrm{ES} \\
6 \mathrm{ES} \\
1 \mathrm{HS} \\
3 \mathrm{HS} \\
3 \mathrm{CHS}\end{array}$ & 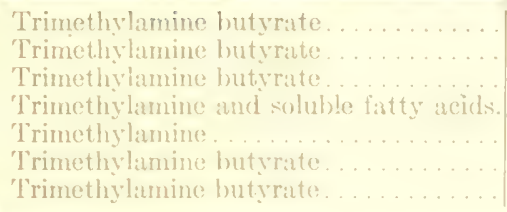 & 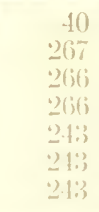 & $\begin{array}{l}\text { Fishy } \\
\text { Fishy, oily } \\
\text { Fishy } \\
\text { Fishy } \\
\text { Fishy, oily } \\
\text { Fishy } \\
\text { Fishy }\end{array}$ & $\begin{array}{l}\text { Fishy } \\
\text { Fishy } \\
\text { Fishy } \\
\text { Fishy } \\
\text { Fishy, tallowy } \\
\text { Fishy } \\
\text { Fishy }\end{array}$ \\
\hline
\end{tabular}

The results obtained from these experiments bring out some very interesting facts. While there are several confleting opinions as to the presenee of the fishy flavor in any particular sample, it is nevertheless evident that the greatest number of positive comments is found in the samples eontaining trimethytumine in one form or another. It will also be notieced that usually the greatest uniformity of such comments is found in the samples containing trimethylanine in unstable form. This is partimlinly true ats to the simmules to which trimethylumine was added alone, in combination with butyre alcid, or in combination with the mixed soluble fatty ateds of hutfer. 'These results atre in harmony with the volitility, the taste, and the odore of the eompounds in pure state. The lack, in a few instaneses, of al majority opinion with regard to the samples containing trimethylumine oleate, trinethylamine stearate, and trimethylanne with the mixed insoluble acids, might be explained on the basis that, sine these substanese werese sextremely matable, the trimethylamine had neaty all volatilized hetore the time of seoring. The greatere number of positive comments from the salted-butter is also worthy of note, and, from what is generally known regarding the oceurrenese of the fishy flavol in such butter, it might tend to strengthen the trimethylumine theory of this flarol. Another foature found in this series of experiments is that at greatere number of fishy-flavored samples were found where the acdity of the arean wats the lowest. This eondition is in hamony with the chemistry involved, for the reason that butter made from low-acid cream contains lest lactic acid when fresh than is 
found in butter made from high-acid cream. It is therefore conceivable that trimethylamine given off by the unstable compounds which were added could not be taken up by the exeess lactic acid in the butter to form the more stable trimethylamine lactate. The finding of numerous fishy-flavored samples where trimethylamine lasetate had been added to low-acid butter might be explained on the basis that eertain conditions were present, possibly particular enzynes, which were eapatble of bringing about the more rapid discociation of the trimethylamine lactate, and that due to the absenere of suflicient free latetic acid to hold the trimethytamine it passed inte the frees state. This explatnation is borne out by the fact that in the butter from high-acid cream there were: at smaller number of samples showing fishiness where the lacetate was added. It might be stated further that one woukl naturally expect to find al greater rariety of enzymes eapable of bringing about the abose-deseribed deromposition in unripened than in ripened eream.

The evidenere obtained frome the various samples of butfer to which trimethylamine butylate was adderl, indientes a striking redationship between this substanes and the fishy flarol. This serems to be true regardless of the type of butter, and to a certain extent regardless of the presenee of sodium chloride. The extemely volatile mature of this

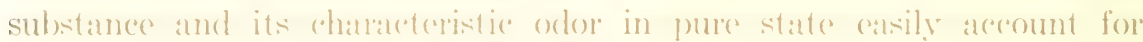
the results obtained. The fate that the we were more positive eromments on the butters enentaining butrite ared alone than there were on butters containing the other acids alone, indieates that this substance may be al contributing fatetor in the deredopment of the fishy flavor under natural conditions. This forture indicates atso that the fishy flator may be due to a definite balane between at deremposition yicdding trinethylamine

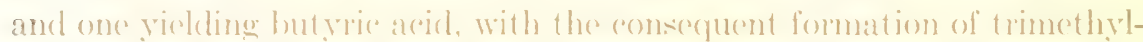
amine butyrate. 'This associative aretion would be entirely posible in storage butter, judging from what is known regarding these formentations.

In the foregoing discussion the writer has ealterl attention to ecrtain theoretial possibilities which might erorelate the findings with what is generally known regarding the varions types of nomal and of fishyflavored butter. It may be satid. howerer', that the evidenee points strongly omated trimethylanine as a contributing factor in fishy-flavored butter. The amount of this substanee responsible for the flaver described as fishy in these experiments is very small. It would be at hazardous 
guess to assign a definite quantity, other than to say that in all cases there was less than 85 parts per million.

\section{QUANTITATIVE ESTIMATION OF TRIMETHYLAMINE IN FISHY BUTTER}

The results obtained by working trimethylamine into normal butter warranted an attempt to isolate this substance from samples of fishyflavored butter found on the market. One of the first difficulties met with in this connecetion was the lack of a method which would aceurately measure the small amounts of trimethylamine that would be found. The method that was finally worked out consisted of a combination and modification of the methods of Folin and Macallum (1912) for ammonia and of Budai [Bauer] (1913) for trimethylamine. The adaptation of these methods for this purpose was as follows:

The material in which trimethylamine and ammonia were to be determined was concentrated to a volume not execeding 15 eubie eentimeters. This material was then placed in the proper tube of the Folin apparatus, 10 grams of anhydrous potassium carbonate was added, and the mixture was covered with a thin layer of kerosene to prevent foaming. This mixture was aspirated for five hours. The ammonia and trimethylamine set free by the potassium earbonate was collected in N/10 hydrochloric acid. The excess acid was titrated with exactly $\mathbf{N} / 100$ alkali, methyl red being used as the indicator. The results of this titration gave the total amount of the mixture of ammonia and trimethylamine. To this titrated mixture 10 cubic centimeters of a neutral 40 -per-eent formaldehyde solution was added. The ammonium chloride present reacted with the formaldehyde to form hydrochlorie acid and hexa-methylene-tetramine according to the equation

$$
6 \mathrm{HCOH} \text { and } 4 \mathrm{NH}_{4} \mathrm{CL}_{2}=\left(\mathrm{CH}_{2}\right)_{1} \mathrm{~N}_{4}+4 \mathrm{HCL} \text {, and } 6 \mathrm{H}_{2} \mathrm{O} \text {. }
$$

The hexa-methylene-tetramine boing neutral, the hydrochlorie acid liberated from the ammonium chloride was titrated and the ammonia was caleulated from this titration. Since the trimethylamine hydrochloride present was not affected by the formaldehyde, the trimethylamine was ealeulated by differences. Since this is essentially a micro method, the technique involved is of the utmost importance. In all cases the volume of the solution to be titrated was kept as nearly constant as possible, and the same amount of indicator was used for each titration. A check on the standard acid and alkali was made with each 
determination, and the curl-points of all neutralization processes were compared colorimetrieally with the standard neutral color. In table 11 are shown the results obtained by this methed from mixtures of known amounts of trimethylanine hychochloride and ammonium chloride:

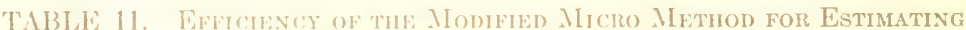

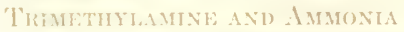

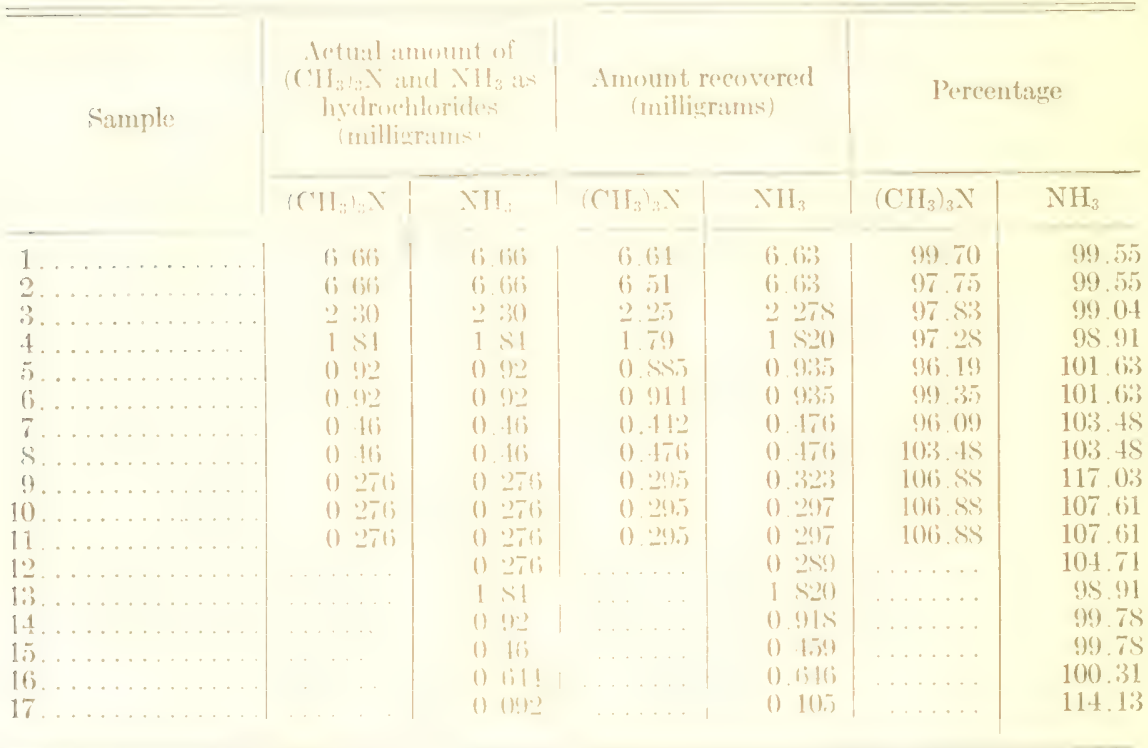

The results shown in table 11 latring justified the reliability of the micro method for motsuring small anounts of trimethylumine, a number of fishy-flavored simmples of humere were subjected to analysis. 'The butter was thomoly wastert in a separatory fumed five times with equal

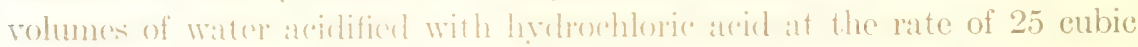
centimeters of nommal areil to the liter. 'The wash water was then evaporated to at small volume as (quickly as possible, and the trimethylamine and ammonia wore determimed as ont lined above.

In table 12 ares shown the trimethylamine and ammonia results obtained from fishy butfer appesuing in commeree and procured from widely different localitios. The ammonial results are shown as a matter of 
interest but they probably have no direct bearing on this particular problem. Since the experiments with artificially produced fishy flavor seemed to indicate the importance of acidity, the acid values of the samples are also included. The acidity is expresed as enbie centimeters of $\mathrm{N} / 10$ sodium hydroxide ueed to neutrilize 20 grams of butter in boiling alcohol. Trimethylamine and ammonia are expresserl in parts per million.

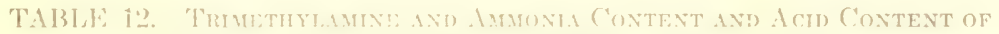

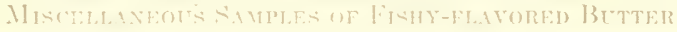

\begin{tabular}{|c|c|c|c|}
\hline stimip)le & $\begin{array}{l}\text { Trimes ly lamine } \\
\text { parts fuer million }\end{array}$ & $\left|\begin{array}{c}\text { Ammonia } \\
\text { (parts per million) }\end{array}\right|$ & $\begin{array}{l}\text { Acid } \\
\text { value }\end{array}$ \\
\hline 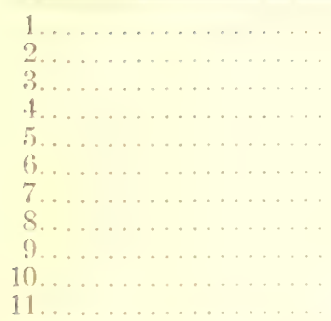 & 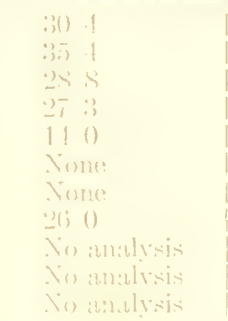 & 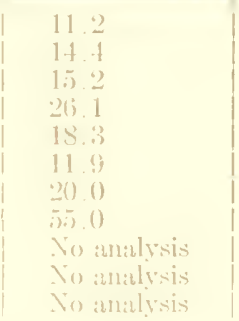 & $\begin{array}{l}3.8 \\
5.7 \\
5.5 \\
5.4 \\
6.8 \\
3.5 \\
2.7 \\
3.8 \\
3.8 \\
3.0 \\
11.0\end{array}$ \\
\hline
\end{tabular}

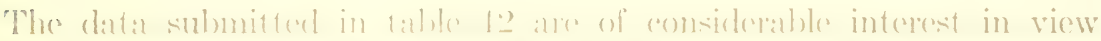
of the history of some of the sumples. ()n arrivinge at the laboratory, all of the simples, with the exerpotion of samplese is and 11 , were seored as fishy hy serecral jucleges. Theres two exerptions were sumples of butter sent from at distance and were prestmathly seored ats tishy whon shipped

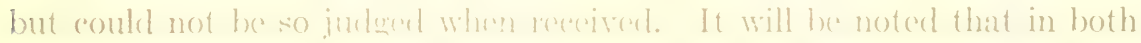
cases there was a highere acid value than in any of the of here sumples, and also that the trimethylumine content of sample is is low. With these

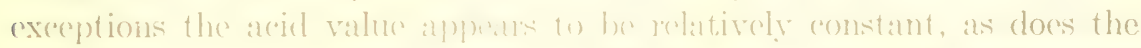
trimethylamine content with the exeeption of simples of and 7 , in which no trimethylamine whaterere wats found. The majority of these results would

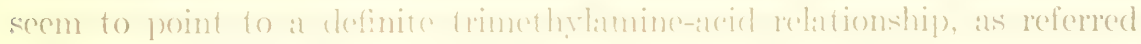
to elsewhere in this palpure. It is to be receretted that in there instances the sample of hutter submittert was foo small to watrant amalysis. The available chata, hemerers. point to trimethytamine ats one of the causal agents in fishy-flawored butter: 


\section{DETELOMIEXT OF FINIY FLAVOR IN EXPERIMENTAL BUTTERS}

In oreter that the development of the fishy flavor might be more carefuily studied. there sories of experimental butters were made with the object of defermining the influenere of pasterurization, of acidity developed Huriug ripeninge of the cream, of ateling lactie acid to the eream, of inoculating butter with lactir-acid bacteriat, and of salt. The procedure folJower in eateh of these series consisted of making nime different types of butere salted and unsalted, from the sume original lot of eream. The different series were mate at interrals of flom there to six werels. The

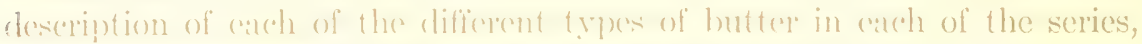
and the designation of the sumples, ate shown in tathe 13:

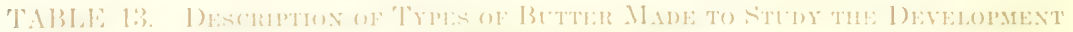

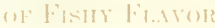

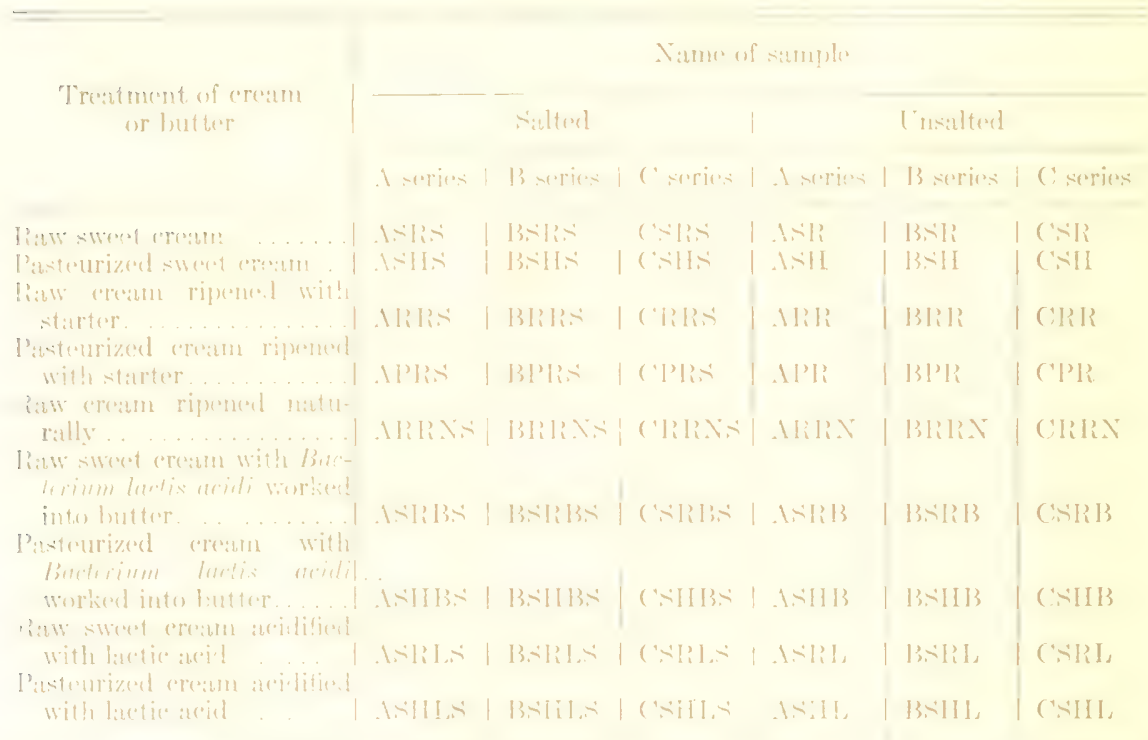

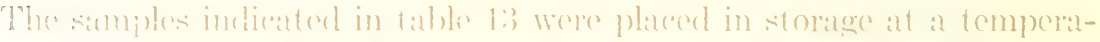

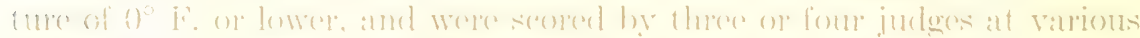
intervals. The results of these seremings atre given in lathle 1.4. Noncharateristic flaver's are purposedy onitted from this table. 
TABTE 14. Comments OF JThefes on the Different Types of Expertmental

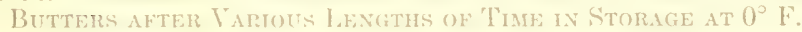

\begin{tabular}{|c|c|c|c|c|c|c|}
\hline \multirow{2}{*}{ Sample } & \multirow{2}{*}{$\begin{array}{c}\text { Acid } \\
\text { in } \\
\text { ('re'ibmt } \\
\text { (per cent) }\end{array}$} & \multirow{2}{*}{$\begin{array}{l}\text { Days } \\
\text { in } \\
\text { storage? }\end{array}$} & \multicolumn{4}{|c|}{ Comments by judges } \\
\hline & & & No. 1 & $X_{0}^{+} \cdot 2$ & No. 3 & $N^{\top}() .1$ \\
\hline 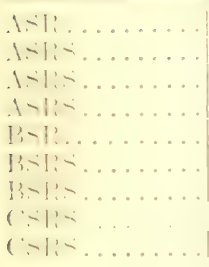 & 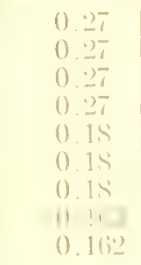 & 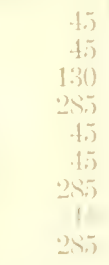 & $\begin{array}{l}\text { lishy } \\
\text { lishy } \\
\text { rishy } \\
\ldots \ldots \ldots \\
\text { ()ily } \\
\ldots \ldots \\
\ldots \ldots\end{array}$ & $\begin{array}{l}\text { Fishy } \\
\ldots \ldots \ldots \\
\ldots \ldots \ldots \\
\ldots \ldots \ldots \\
\ldots \ldots\end{array}$ & $\begin{array}{l}\text { lishy } \\
\text { ()ily } \\
\text { ()ily } \\
\ldots . . \\
\text { Jishy }\end{array}$ & Fishy \\
\hline $\begin{array}{l}\text { IBSIIS . . . . . } \\
(-11 \ldots \ldots \\
\text { CSII } \ldots \ldots \ldots\end{array}$ & $\begin{array}{l}0.16 \\
0.111 \\
0.111\end{array}$ & $\begin{array}{l}1,3 \\
1,30 \\
1,3\end{array}$ & $\begin{array}{l}\text { Miotallic: } \\
\text { Mictallio: }\end{array}$ & (oily & $\begin{array}{l}\text { Mletiullie } \\
\ldots \ldots \ldots \\
\ldots \ldots \ldots\end{array}$ & $\begin{array}{l}\ldots \ldots \\
\ldots \ldots \\
\ldots \ldots\end{array}$ \\
\hline 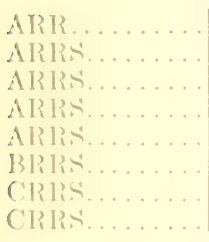 & 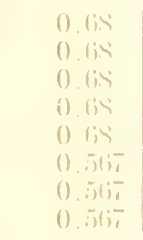 & $\begin{array}{r}4.5 \\
15 \\
90 \\
130 \\
25,5 \\
1.5 \\
(10) \\
24.5\end{array}$ & 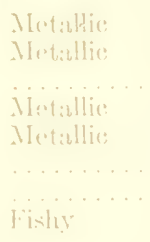 & $\begin{array}{l}\text { Metallie } \\
\text { Mretallic } \\
\text { (Jily } \\
\text { Metallie: } \\
\text { Mrenallic: } \\
\text { Mrotallice } \\
\text { ()ily }\end{array}$ & 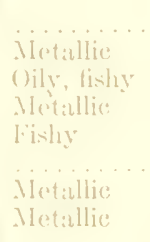 & $\begin{array}{l}\ldots \ldots \ldots \\
\ldots \ldots \ldots \\
\ldots \ldots \\
\ldots \ldots \\
\ldots \ldots \\
\ldots \ldots \\
\text { lishy }\end{array}$ \\
\hline 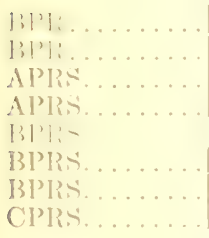 & 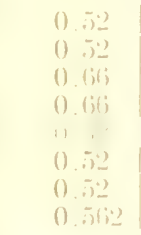 & 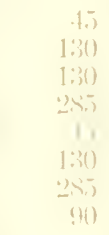 & $\begin{array}{l}\text { MLetizllic: } \\
\text { ()ily }\end{array}$ & 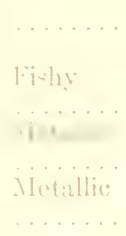 & $\begin{array}{l}\text { ()ily } \\
\text { Mletallie } \\
\ldots \ldots \\
\ldots \ldots \\
\text { ()ily }\end{array}$ & $\begin{array}{l}\text { Hishy } \\
\ldots \ldots \\
\ldots \ldots \ldots \\
\ldots \ldots \ldots\end{array}$ \\
\hline 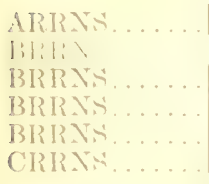 & 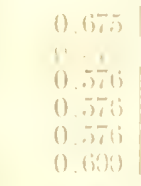 & $\begin{array}{l}1: 3(1) \\
1 \\
15 \\
(y) \\
205 \\
3(y)\end{array}$ & $\begin{array}{l}\text { ()ily, fishy } \\
\text { firlyy } \\
\text { lishy } \\
\text { lishy }\end{array}$ & $\begin{array}{l}\text { Pishy } \\
\text { linhy } \\
\ldots \ldots\end{array}$ & 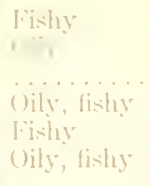 & $\begin{array}{l}\ldots \ldots \\
\ldots \ldots \ldots \\
() \text { ily }_{y} \\
\ldots \ldots \\
\ldots\end{array}$ \\
\hline 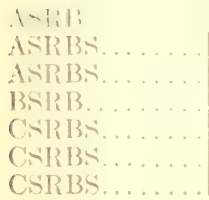 & 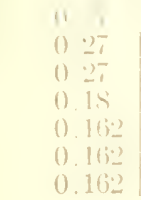 & $\begin{array}{r}1 \\
45 \\
130 \\
45 \\
1.5 \\
9(1) \\
25.5\end{array}$ & $\begin{array}{l}\text { lishy } \\
\text { lishy } \\
\text { ()ily, fishy } \\
\ldots \ldots \ldots \\
\ldots \ldots \ldots \ldots\end{array}$ & $\begin{array}{l}\text { Fishy } \\
\text { Fishy } \\
\text { Oily.... }\end{array}$ & $\begin{array}{l}\ldots \ldots \ldots \\
\text { (jily } \\
\text { (j)ily } \\
\text { Mletallic }\end{array}$ & $\begin{array}{l}\ldots \ldots \\
\ldots \ldots \\
\ldots \ldots \\
\ldots \ldots \\
\ldots \ldots \\
\ldots \ldots\end{array}$ \\
\hline
\end{tabular}


T.1BLLE: 1.1 (conctuded)

\begin{tabular}{|c|c|c|c|c|c|c|}
\hline \multirow{2}{*}{ Sample } & \multirow{2}{*}{$\begin{array}{l}\text { Arid } \\
\text { in } \\
\text { cre:tm } \\
\text { (per exent) }\end{array}$} & \multirow{2}{*}{$\begin{array}{c}\text { 1)ins } \\
\text { in } \\
\text { storizese }\end{array}$} & \multicolumn{4}{|c|}{ Comments by juderes } \\
\hline & & & No. 1 & No. 2 & No. 3 & No. 4 \\
\hline 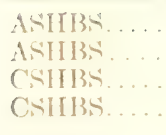 & $\begin{array}{l}\text { 0) } 1 \leqslant ? \\
\text { () } 1 \leqslant ! \\
\text { () } 1.11 \\
\text { () } 1.11\end{array}$ & $\begin{array}{r}130 \\
25.7 \\
1.7 \\
90\end{array}$ & $\begin{array}{l}\text { Fishy } \\
\text { Metallic } \\
\text { Metallic }\end{array}$ & $\begin{array}{l}\text { Oily } \\
\text { Metallie } \\
\ldots \ldots \ldots\end{array}$ & $\begin{array}{l}\text { Iishy } \\
\text { Metallic } \\
\ldots \ldots \ldots\end{array}$ & $\begin{array}{l}\text { Metallic } \\
\ldots \ldots \ldots \\
\ldots \ldots \ldots\end{array}$ \\
\hline 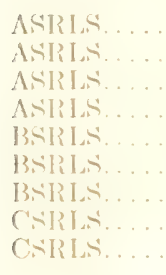 & 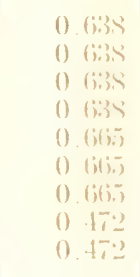 & 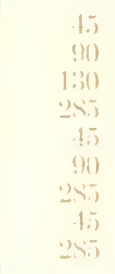 & $\begin{array}{l}\text { rishy } \\
\ldots \ldots \ldots\end{array}$ & $\begin{array}{l}\text { Pishy } \\
\text { Fishy } \\
\text { liishy } \\
\text { lishy } \\
\text { loishy } \\
\text { Mettillie } \\
\text { Motiallie }\end{array}$ & $\begin{array}{l}\text { Fishy } \\
\text { Fishy } \\
\text { Fishy } \\
\ldots \ldots \\
\text { Fishy } \\
\text { Wishy }\end{array}$ & $\begin{array}{l}\ldots \ldots \ldots \\
\text { Fishy } \\
\ldots \ldots \ldots \\
\text { Metallic } \\
\text { Oily }\end{array}$ \\
\hline 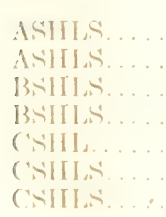 & 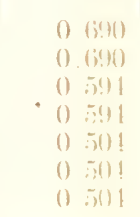 & $\begin{array}{l}13010 \\
285 \\
1.5 \\
90 \\
15 \\
1.5 \\
2 \times 5\end{array}$ & $\begin{array}{l}\text { Wishy } \\
\text { Fishy } \\
\text { Fishy }\end{array}$ & $\begin{array}{l}\text { Whatulic } \\
\text { Fishy } \\
\text { ()ily } \\
\text { rishy }\end{array}$ & $\begin{array}{l}\text { Motallie } \\
\text { lishy } \\
\ldots \ldots \ldots \\
\ldots \ldots \ldots \\
\ldots \ldots \ldots \\
\text { lishy }\end{array}$ & Oily \\
\hline
\end{tabular}

In eonsidering the pexults from the different types of experimental butter, it is evident that there is considerathle dirersity of opinion among the judges as to the presenere or the absenere of the fishy flater in certain

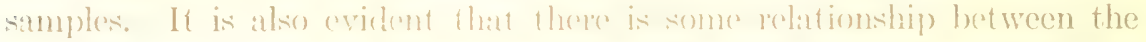
metallice, oily, and fishy flavol's, particulary when these flavors are not suffieiently pronounered to be distinetive ats wats the catse in these samples.

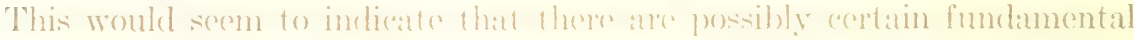
conditions which are eommon to the derelopmenent of each of these flavors.

Even tho there is difference of opinion at to the presenee of the charanderistic flavors, centain conclusions may be drawn from these experiments. Probably one of the most signifieant is the presenese of the fishy ore the metallie flavor in the salted butters. ()f a total of 10.5 characteristic eomments, 9.3 are found in the simmples antatning salt. Another eonchusion which may les drawn from the relative agreement of the fudges, is that the fishy flavor appears oftener in the butter made from 
high-acid cream than in that from low-acid arom, there being little differenee whethere the areit was deredopert by the use of starter, by ripening naturally, or by the ardition of lacetic acid to raw sweet cream.

These findings, compaterl with the results obtained from pasteurized

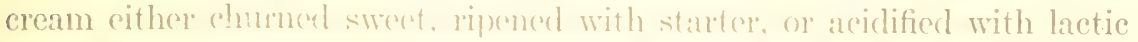
acid - all of which showed fower fishy simples than did raw eream - elearly indieate that the fundinment muse of this butter defeet is primarily biological, not brought about hy at spontaneons chemieal change in which such agenerese do not play a part. White it is orident that acid plays an important role in the deredepment of the fishy flaver, it is equally elear that there ane other important contributing factors. Just what these factors are, is monkown. The variable results obtained from the same type of butter in the different series would indieate that the original

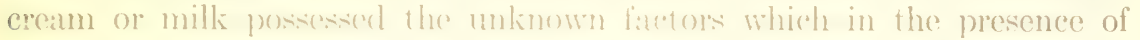
lactie acid determined the dexelomment of the flavers. From the faret

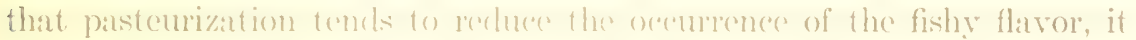
is quite probable that these aceneses are bacterial anzymes which are only partially inacetivated he heat; or it may even be possible that certain microorganisms which are incerporated in the hutter from the eream,

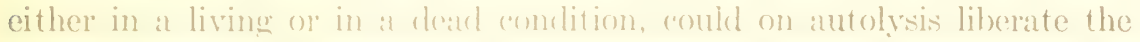
enzymes rapathle of smpulying the determining fatetor. It may also be added that pustentization may kill eortain emzymes and not other's, the particular ones that are impertant heing amonge those killed.

These contentions are further supported by the faret that in the butters made from raw sweret cream there is a stegerstion of fishiness after the first storage perient which is not fomml aftere the lomger periods, the disappearanee or latek of furthere alevelopment of the flatror being due to the absenee of the propere actid condition. It is alear that larege number's of

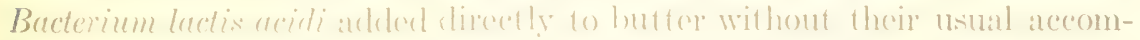
panying by-products are not the cauce of any characteristic change in flavor.

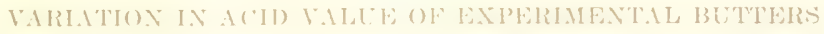

The importanere of acedity in the manifestation of the fishy flavor by trimethylamine, and the reditively eonstant ateicl value of the miscellaneous samples of fishy butter found on the market. amphasized the importance of studying this fuctur in the experimental butters described 
above. The variation in acid value of the different types of butter in each of the three series is shown in tables 15, 16, and 17 . Results are expressed as enthice rentimeter's of 1 T 10 alkiali necessary to neutralize 20 grams of butter in boiling nentral alcohol.

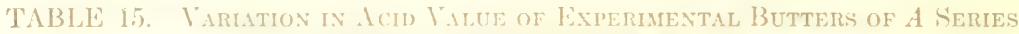

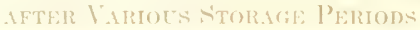

\begin{tabular}{|c|c|c|c|c|}
\hline \multirow{2}{*}{ Simple } & \multicolumn{4}{|c|}{ Acid value aftor various storage periods } \\
\hline & 1:3 day: & Sf ditys & 128 days & 310 days \\
\hline $\begin{array}{l}\text { AsR } \ldots \ldots \ldots \ldots \ldots \\
\text { ASTR . . . . }\end{array}$ & $\begin{array}{l}8.8 \\
7.4\end{array}$ & $\begin{array}{r}10.5 \\
8.0\end{array}$ & $\begin{array}{r}10.8 \\
8.2\end{array}$ & $\begin{array}{l}13.0 \\
10.0\end{array}$ \\
\hline 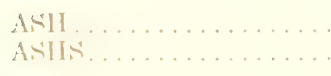 & 5.8 & 8.6 & $\begin{array}{r}10.0 \\
5.7\end{array}$ & $\begin{array}{r}12.1 \\
7.2\end{array}$ \\
\hline $\begin{array}{l}\text { ARR . } \\
\text { ARRiS . }\end{array}$ & $\begin{array}{ll}8.2 & 1 \\
8.7 & 1\end{array}$ & 8.5 & $\begin{array}{l}9.3 \\
8.6\end{array}$ & $\begin{array}{l}10.3 \\
10.3\end{array}$ \\
\hline $\begin{array}{l}\text { Al'R } \ldots \ldots \ldots \ldots \\
\text { Al'lis } \ldots \ldots \ldots \ldots\end{array}$ & \begin{tabular}{l|}
7.7 \\
8.1
\end{tabular} & $\begin{array}{l}7.9 \\
7.8\end{array}$ & $\begin{array}{l}8.2 \\
8.0\end{array}$ & $\begin{array}{l}9.8 \\
9.6 \mathrm{i}\end{array}$ \\
\hline $\begin{array}{l}\text { ARRN } \ldots \\
\text { IRRIS }\end{array}$ & $\begin{array}{l}8.9 \\
8.81\end{array}$ & 86 & 8.2 & $\begin{array}{l}10.5 \\
10.5\end{array}$ \\
\hline 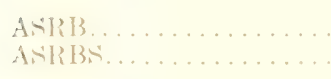 & $\begin{array}{l}9.71 \\
7.11\end{array}$ & $\frac{10.1}{7.7}$ & $\frac{11}{7} \cdot \frac{2}{4}$ & $\begin{array}{r}15.0 \\
9.8\end{array}$ \\
\hline 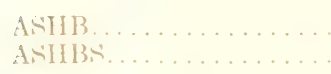 & $\begin{array}{l}5.7 \\
5.8\end{array}$ & $\begin{array}{l}S 6 \\
5 . !\end{array}$ & $\begin{array}{l}0.5 \\
5.8\end{array}$ & $\begin{array}{r}14.3 \\
9.7\end{array}$ \\
\hline $\begin{array}{l}\text { ANRI . . } \\
\text { ASIILS.. }\end{array}$ & $\begin{array}{l}8 \% \\
89\end{array}$ & $\begin{array}{l}8.8 \\
8.9\end{array}$ & $\begin{array}{l}\text { S.S } \\
\text { 9. } .0\end{array}$ & $\begin{array}{l}10.3 \\
10.0\end{array}$ \\
\hline $\begin{array}{l}\text { AsHI } \ldots \\
\text { ANHL . . }\end{array}$ & $\begin{array}{l}70 \\
7 \\
7\end{array}$ & $\begin{array}{l}7.1 \\
0.5\end{array}$ & $\frac{7.2}{7.1}$ & $\begin{array}{l}8.0 \\
8.5\end{array}$ \\
\hline
\end{tabular}

The datal presented in tables 15, 16, and 17 , show many interesting features, some of which ane worthy of clisenssion in enneetion with this preblem. It maty be stated in the begimning that the variations in acid value of the different types of butser point to biologieal agencies as the camese of these variations. The lowere ated vatue obtained in nearly all instances from salted butter indieates a preservative aletion by the salt, a function which is well known. The greatest increase in acidity is 
shown in the butter made from raw swect cream. It is interesting to note that very few of the samples were scored as fishy. When such a condition was suggested, it is to be noted that it oceurred after the first storage period, when the acid value was lowest.

Table 16. Variation in Acid Value of Expermental Butters of B Series after Vartous Storage Periods

\begin{tabular}{|c|c|c|c|c|}
\hline \multirow{2}{*}{ Simple } & \multicolumn{4}{|c|}{ Acid value after various storage periods } \\
\hline & 20 days & SS days & 126 days & 286 days \\
\hline 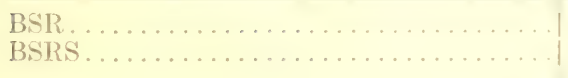 & $\begin{array}{r}13.9 \\
9.7\end{array}$ & $\begin{array}{r}14.6 \\
9.5\end{array}$ & $\begin{array}{l}15.2 \\
10.0\end{array}$ & $\begin{array}{l}17.5 \\
10.6\end{array}$ \\
\hline 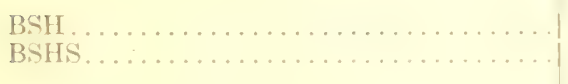 & $\begin{array}{l}7.3 \\
6.3\end{array}$ & 9.0 & $\begin{array}{r}10.8 \\
6.8\end{array}$ & $\begin{array}{r}13 \cdot 4 \\
7.6\end{array}$ \\
\hline 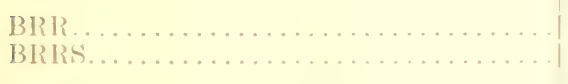 & $\begin{array}{l}10.1 \\
10.1\end{array}$ & $\begin{array}{l}10.2 \\
10.1\end{array}$ & $\begin{array}{ll}10 & 8 \\
10 & 4\end{array} \mid$ & $\begin{array}{l}11.9 \\
11.3\end{array}$ \\
\hline 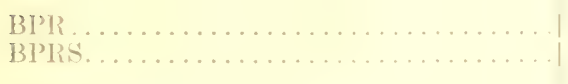 & $\begin{array}{r}10.1 \\
9.8\end{array}$ & $\begin{array}{l}10.1 \\
10.1\end{array}$ & $\begin{array}{r}10.0 \\
9.0\end{array}$ & 11.2 \\
\hline $\begin{array}{l}\operatorname{BRRN} \ldots \ldots \ldots \\
\text { BRRNS } \ldots \ldots \ldots\end{array}$ & $\begin{array}{l}11.3 \\
11.2\end{array}$ & 11.7 & $\begin{array}{l}12.6 \\
11.8\end{array}$ & $\begin{array}{l}1.1 .3 \\
13.8\end{array}$ \\
\hline 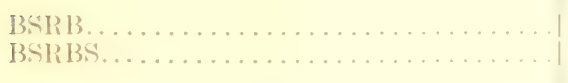 & $\begin{array}{l}13.6 \\
10.6\end{array}$ & $\begin{array}{l}13.5 \\
10.8\end{array}$ & $\begin{array}{l}1.1: 3 \\
10: 5\end{array}$ & $\begin{array}{l}15.1 \\
12.0\end{array}$ \\
\hline 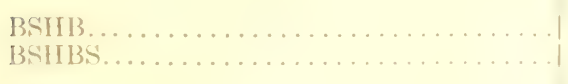 & 8.1 & $\begin{array}{l}8.4 \\
6.6\end{array}$ & $\begin{array}{l}9.0 \\
\text { (j. } 8\end{array}$ & $\frac{9.2}{8.0}$ \\
\hline 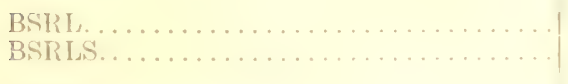 & $11 . \overline{5}$ & $\begin{array}{l}11.5 \\
11.1\end{array}$ & $\begin{array}{l}11.6 \\
11.6\end{array}$ & $\begin{array}{l}12.9 \\
12.6\end{array}$ \\
\hline 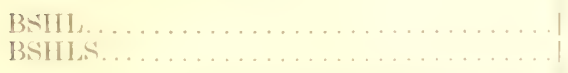 & $\begin{array}{l}9.41 \\
(1) .5\end{array}$ & $\begin{array}{l}9.4 \\
9.6\end{array}$ & 9.5 & $\begin{array}{l}11.0 \\
10.1\end{array}$ \\
\hline
\end{tabular}

With reference to the samples from pasteurized cream as compared with those from law sweet eream, it will be notieed that pastemization has tended to cause a lowering of the acid value but has not entirely prevented its gradual inerease. This would be in aceord with possibilities already stated regarding batederial engymes. The data show also a retarding action exerted by the aced originally in the cream. This is evident in the butter made from both raw and pasteurized eream ripened 
with starter, from maw cromm ripenert mathatly, and from both raw and

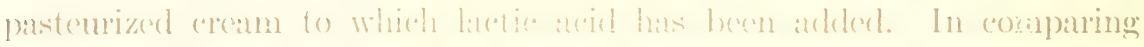

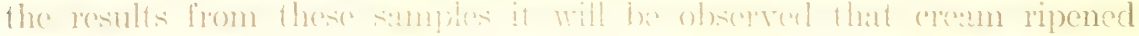

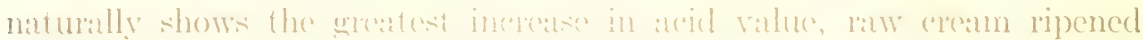

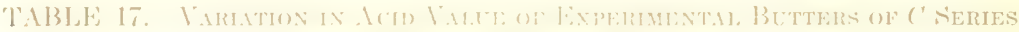

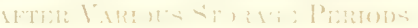

Simple.

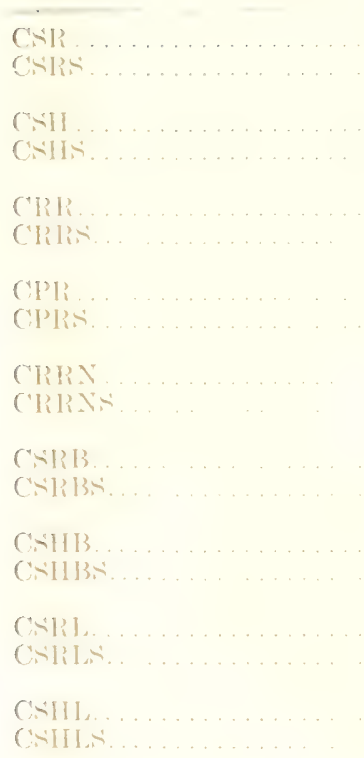

Arid valne atter varions storuge periods

\begin{tabular}{|c|c|c|c|}
\hline (iacess ? & Is lays & 00 diuys & $272 \mathrm{days}$ \\
\hline$\because \therefore 1$ & 10.4 & 11.0 & 13. \\
\hline 741 & 7.9 & 8.0 & 9 \\
\hline 1.1 & 5.6 & 11.0 & 12.0 \\
\hline 11 & 1.7 & 4.4 & 5 \\
\hline 7.61 & (1) $: 3$ & 10). 1 & 12 \\
\hline 7.51 & $\therefore .2$ & 8.1 & 10 \\
\hline 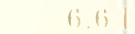 & 7.5 & 8.9 & 10) \\
\hline$(i . j)$ & 7.0 & 7.0 & $s$ \\
\hline 8.7 & (1) 5 & 10.0 & 12 \\
\hline$\therefore \because$ & !) () & (3). 1 & 11. \\
\hline $7 k$ & 10.21 & 11.8 & 13 \\
\hline (i) $!$ & 7.4 & 7.9 & 9.0 \\
\hline$+1+1$ & 6.5 & 7.7 & \\
\hline 1.1 & 4.1 & 1.1 & \\
\hline$\therefore: 3$ & $\therefore i$ & $8.1)$ & 9 \\
\hline$\therefore 1$ & $(3,5)$ & 8.1 & \\
\hline$\therefore \therefore 1$ & $(i, 1)$ & 5.9 & 6.7 \\
\hline (i) (1) & i. 7 & 5.6 & \\
\hline
\end{tabular}

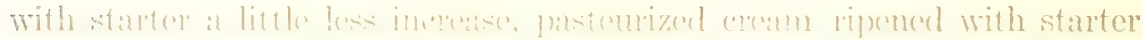

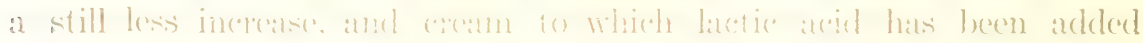

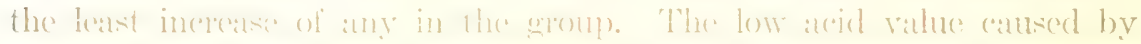

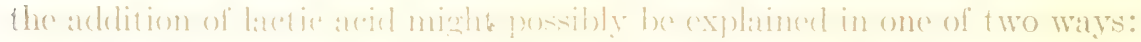

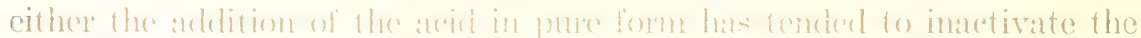

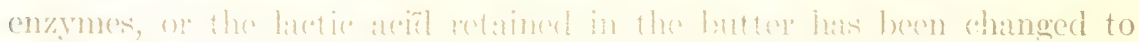

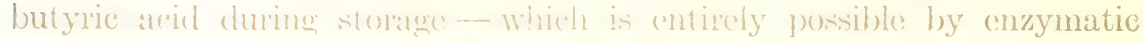


action. If such a change as the latter dict take place, a lowering of the acid value would be manifestert beranse of the formation of a weaker acid which probably has resulterl from the splitting and condensation of two parts of the stronger lactice acid. The posibility of butrrie acid being formed in this way might he smpupenter by the fact that the other samples from high-acid cream showed a lowere acid value than those from sweet aream but hat a higher value tham those to which pure lactic

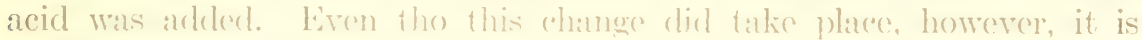
improbable that it could ontirely aterount for the low value indieated. It is more probable that the latetie acid atets as an inhibiting agent. A study of the tables will shew that the increate in ared value of the sour-

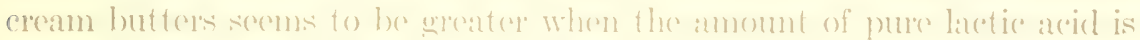

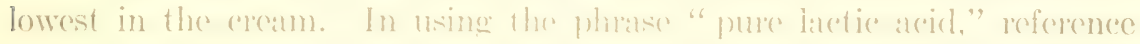
is made to that which wats actiled and also to that deredoperd by baceteria,

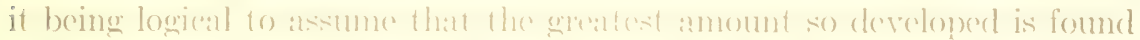

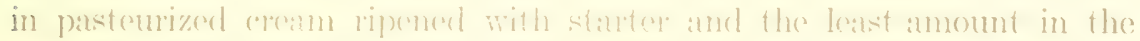

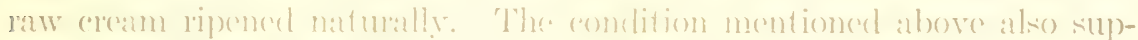

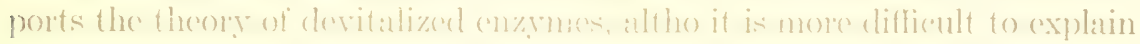

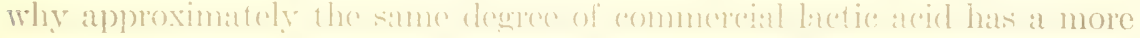

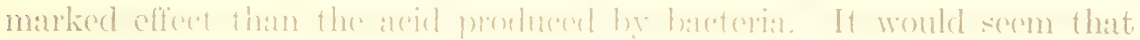

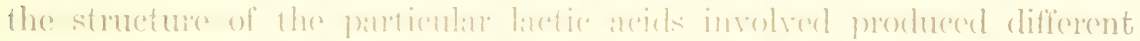

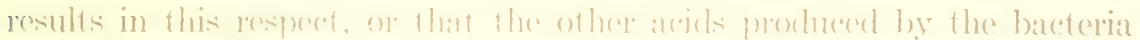
are lessinhibitive than the lase ine.

Regardess of what the explanation for the variatione may he, the data

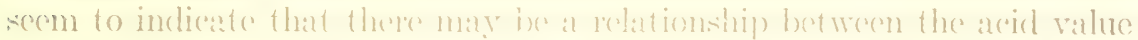

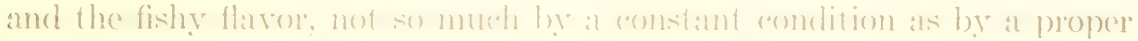

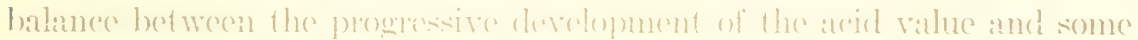

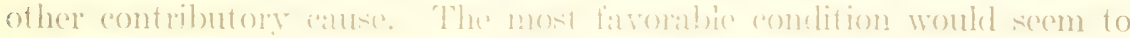

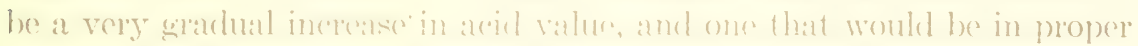
harmony and redationship) to some of hee important and transient fatetor.

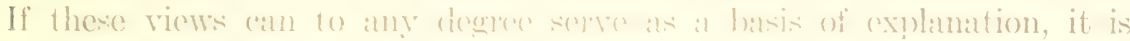

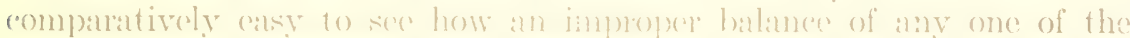

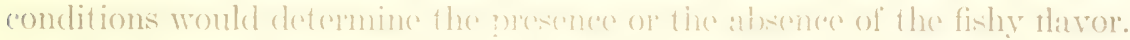
It might also be concerivathe that the internity of the true flatror would

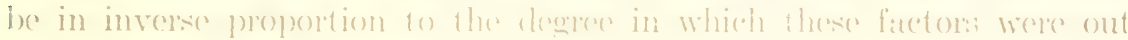

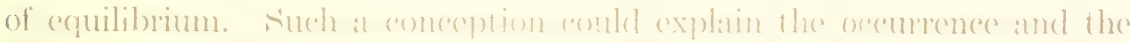
disappearances of the flaver in the sume sample of butter at different 
times, why the fishy, the motallice and the oily flavors sem to be closely

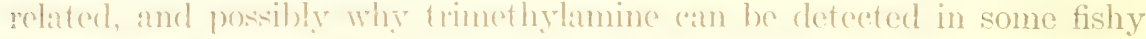
butters and not in of hers.

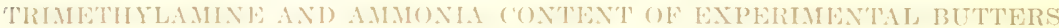

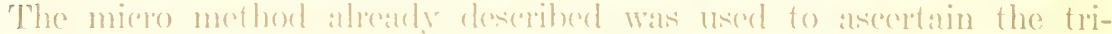
mothylamine and ammonia comtent in the (xperimental butters after

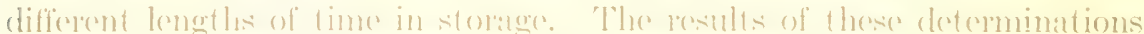

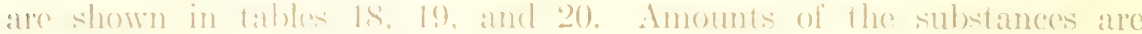
(x)

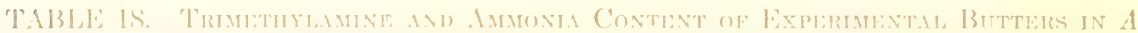

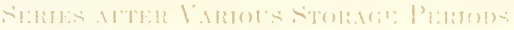

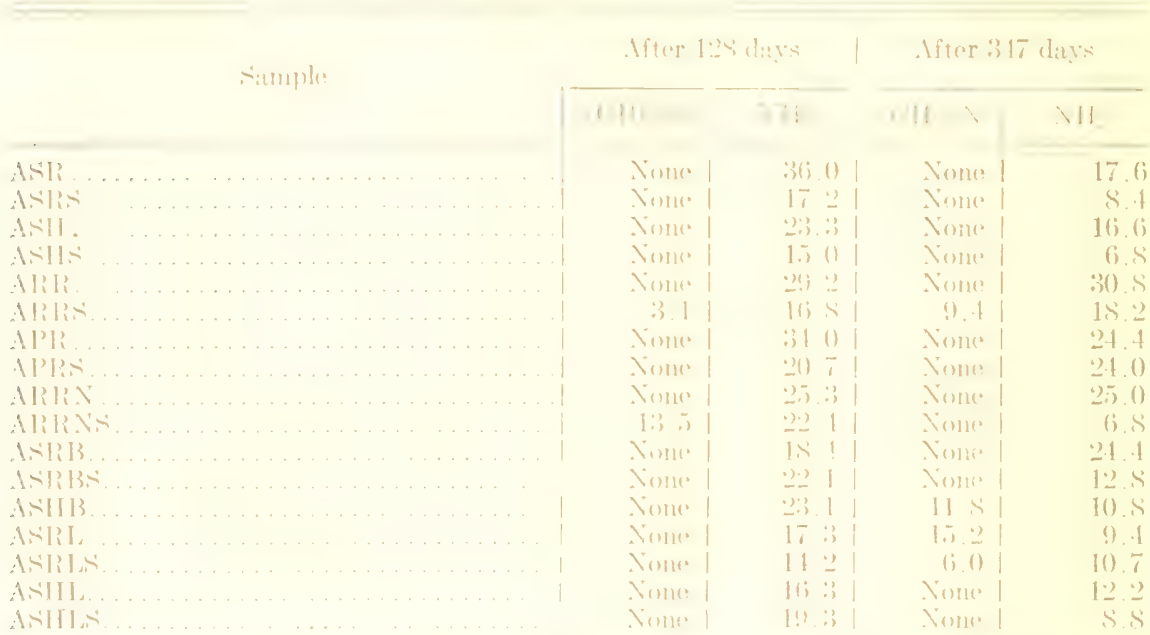

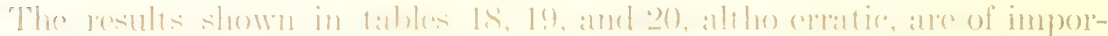
fance ats indieatine the variations in decomposition in the same and in

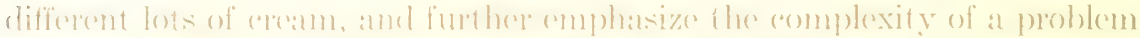

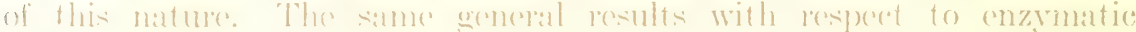

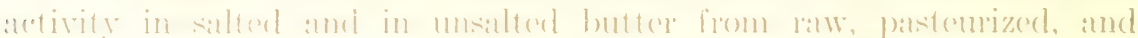

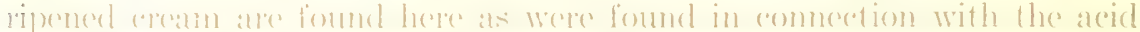

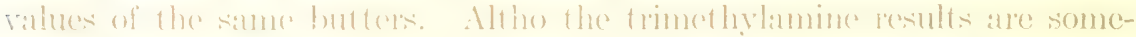
what diseordint, at fondeney is shown for the presenee of this substance 
to harmonize with the samples seored as fishy, metallic, or oily. Of 21 samples, in which trimethylamine was found, 15 were assigned one of the characteristie flavor's by one or more of the judges at some time during the storage period. In 5 of the remaining instances, it is to be noted that, while trimethylamine wats found in the sume type of butter of the same series, its presenee did not hamonize with the characteristic flavor in the salted or the malted sample. On the ofther hand, there were 10 samples of different types of butter which were indieated as having a characteristic flatror by one or more of the judges at some time during the storage period, in which trimethylumine could not be detected.

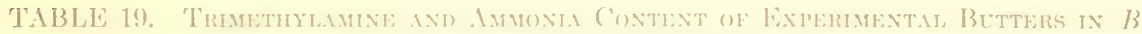

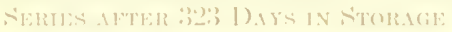

\section{Sitminle}

BSTR.

lisil

BSHS

IBRl?

BRRR

BPI?

BPIRS

BRRR

Blilinis

BNIR13.

BSRBS

BSIIB

BSHBS

BSIII.

BSIRIN

Bsilt.

BSIIL
$\left(\mathrm{CH}_{3}\right)_{3 \times} \mathrm{N} \mid \quad \mathrm{NH}_{3}$

\begin{tabular}{r|r} 
None & 35.2 \\
None & 18.0 \\
530 & 10.2 \\
Yone & 32.8 \\
8.2 & 21.6 \\
10.6 & 23.8 \\
9.4 & 25.0 \\
4.7 & 27.0 \\
None & 32.2 \\
35.4 & 29.4 \\
None & 20.0 \\
5.9 & 223.0 \\
None & 10.4 \\
None & 223.0 \\
None & 17.0 \\
None & 7.4 \\
None & 11.4 \\
&
\end{tabular}

Thile these results are not absolutely andesive, there is nevertheless an indication that trimethylamine may he ono of the contributing factors in the derelopment of the true fishy flaser. It has been shown that

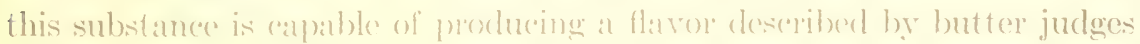

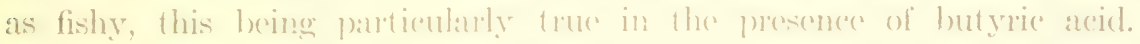
Furthemore, it has beren shown that trimethylamine may be present in fishy-flavored butter. 'Therefore it would mot apprar to be beyond the 
realm of possibility that the results shown in this paper point to a definite trimethylanine and acid relationship ats being the cause of that flavor in butter which resembles the flavor of herring or mackerel brine, and that non-typical flavors resembling other fish proctuets, or the metallic

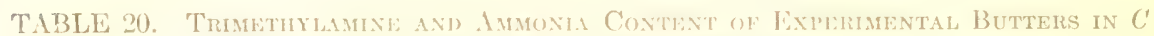

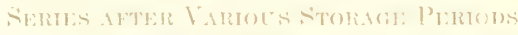

\begin{tabular}{|c|c|c|c|c|}
\hline \multirow{2}{*}{ Sample } & \multicolumn{2}{|c|}{ After 190 dluys } & \multicolumn{2}{|c|}{ ifter 310 days } \\
\hline & & $x: ?$ & $\cdots: 1$ & $11 !$ \\
\hline 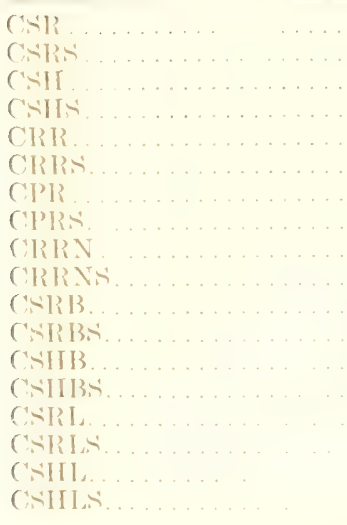 & 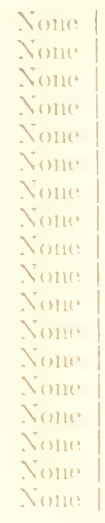 & 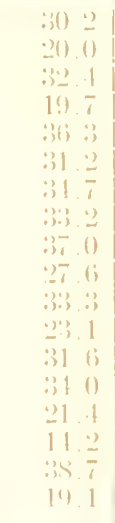 & 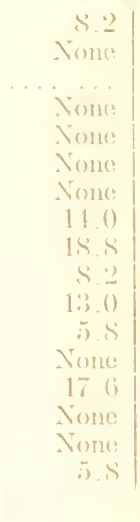 & $\begin{array}{l}33.2 \\
25.2 \\
1.12 \\
20.6 \\
36.2 \\
29.2 \\
27.1 \\
31.8 \\
29.5 \\
33.6 \\
15.6 \\
31.6 \\
13.4 \\
1.8 \\
10.8 \\
20.6\end{array}$ \\
\hline
\end{tabular}

and the oily flavor, mar ha due to an unbalaneing of this relationship, the oceurrence of these flatrors bainge due to factors in which the presence of trimethylamine in (letectable amounts is in no way (ontributery.

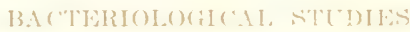

In an effort to correlate the preending observations with the biologieal asperets of the problem, eertain bacteriolegendent studies were carried out. These includerl a batederial andysis of fishy-and non-fishy-flurored butters, and inoculations with pure and mixed (ultures inte) choline, lecithin, butterfat, and cream for the purpose of funding, if possible, an organism or a group of organisms which in some way might contribute to the development of the fisliy flavor. 


\section{Bacterial analysis}

Bacteriological examinations of fishy- and of normal-flavored butter from various sourees seemed to show no characteristic differences in flora, neither were the quantitative results ennistent. Fven tho the samples examined did not alppear to possess marked differenees in flora, cultures of the predominating type were isolated from the fishy samples for the purpose of dotermining a condition under which they might eontribute to the chameteristic flavor. The types of bacteria found included a number of acist-producing rarieties, both coecus and rod forms. Among the species commonly found were Hierococtus lactis acidi, Mic. lactis albidus, Bacterium lactis brevis, Bact. aerogenes, and Bact. lact is flocculus.

The bacteria content of ecrtain samples of fishy- and of non-fishyflavored butter is given in table 21 :

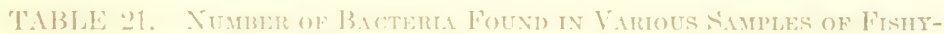

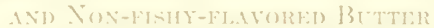

\begin{tabular}{|c|c|c|}
\hline S:tuple & $\begin{array}{c}\text { Character } \\
\text { of } \\
\text { flaver }\end{array}$ & $\begin{array}{l}\text { Bacteria } \\
\text { per } \\
\text { gram }\end{array}$ \\
\hline 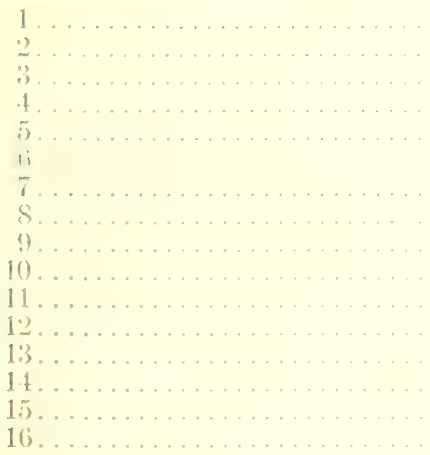 & 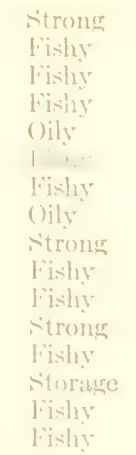 & $\begin{array}{r}1,200,000 \\
1,100,0000 \\
8,000,000 \\
21,600,(000 \\
30,000,000 \\
1,1,16111 \\
13,0000 \\
3,900,000 \\
760,000 \\
23,500 \\
8,300,000 \\
1,600,0000 \\
310,0000 \\
2,600 \\
350,000 \\
46 ., 000\end{array}$ \\
\hline
\end{tabular}

Inoculations for the purpose of dereloping the fishy fletror

The results of previous investigntions show that little suecess has been attained in the attempt to clexelop the fishy flaror in butter by inoculating the butter itself or by inomalating the cream just prior to churning. If the enzymatic idea as already stated in this paper is to be 
upheld, such negative results an be explained by the face that organisms inoculated into the eream just prior to chuming, or into the butter, do not have the opportunity for growth and consecunent production of by-products which they woukl have if allowed to grow in the milk or the cream bofore it is marle into butter. There seems to be wood evidence that there is a rapid dying-off of the bacteria in butter after the first few days of storage. Furthemore, it is well known that low storage temperatures do not entirely prevent maynatic aretivity. Rogers (1909) shows that, while low femperatures delay the development of the fishy flavor, they do not entirely prevent it. In acerortanee with these facts it is conceivable that certain enzymes whing would be produced by the growth of organisms in the cream, and carrien ints the butter, would there continue their aretivity, with the conseruent manifestation of certain

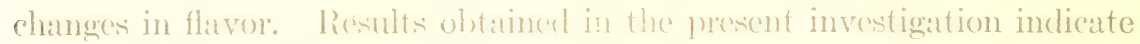
also the importane of at clefinite atein relationship. With these factors in mind, the incendation experiments undertaken in commetion with this problem were carried ont in a menner that would allow for the manifestation of the possibilities indicatcul.

Nine orgunimst all of which were irelated lirom samples of fishy butter and Bacterium ichthyosmius - which Hitmmas (19)17) found would produce the fishy flavor in milk - vire uevel in these experinents. Thes same original lot of cream wats divicied inte six prerts, and cach of these parts was further divider into fon parts, eateh of which was inoculated with a specific organism. Different methoris of hambling the six groups of ten inoculations weh were carried ont in such a way that the effect of acid in ronjunction with the specifie orgunism enulel be determined. Adequate chereks were made fromi uninoentated cream. Pasteurized sweet eream was used as the basis for all inoentations. When the cream was neutralized

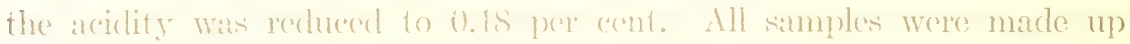
both salted and unsalterl, and were serored after 2.31 diess in storage at a temperature of $0^{\circ} \mathrm{F}$. or lotwer. The presultis of the experiment als regateds walted butter atre given in tablis w2.

It is chatacteristice of the results of this experiment that none of the sumples of unsalted butter shomed any of the chapactopistie flayors and

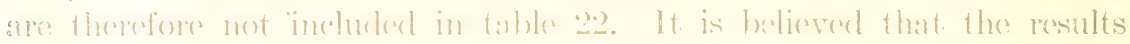
slown in the tathe elenty confinn the opinion that biologieal agencies, particularly baterial anzymes, are respomsible to at arreat degree for the flavors indicated; also, that the fundamental condition necessary for the 
TABle 22. Effect on the Flavor of Salted Buton, of Inoculativg Crean with Specific Organisms under Different Conditions

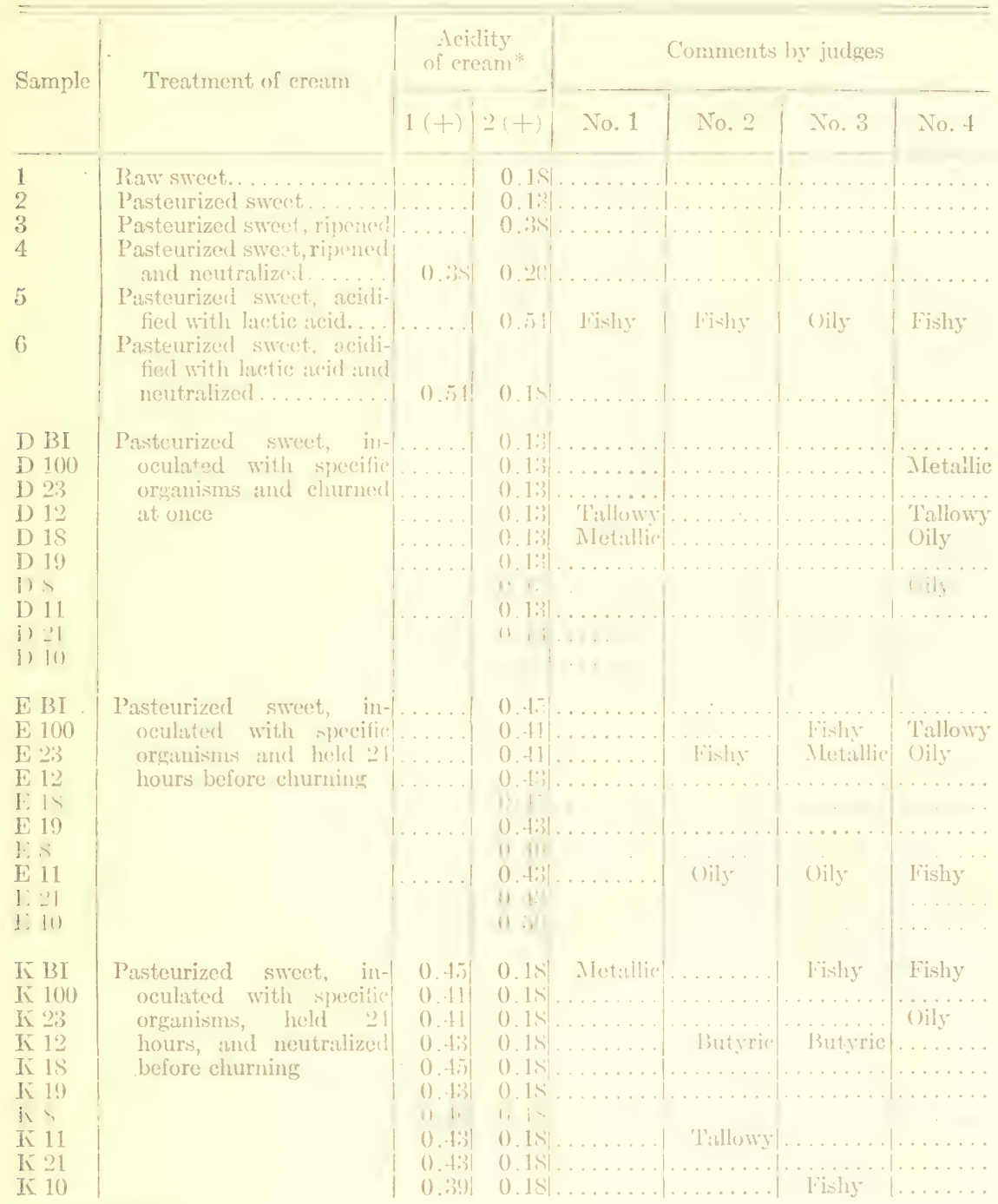

$1(+)=$ acidity at time of neutralizing.

$2(+)=$ acidity at time of churning. 
ILIBTE :-2 (coneluderl)

\begin{tabular}{|c|c|c|c|c|c|c|c|}
\hline \multirow{2}{*}{ Sample } & \multirow{2}{*}{ Troatment of eream } & \multicolumn{2}{|c|}{$\begin{array}{l}\text { Acidity } \\
\text { of ereant }\end{array}$} & \multicolumn{4}{|c|}{ Comments by judges } \\
\hline & & $i(+) !$ & $1(+)$ & No. 1 & $X_{0}, 2$ & No. 3 & No. 4 \\
\hline $\begin{array}{ll}N & B 1 \\
N & 100 \\
N & 23 \\
N & 12 \\
N & 18 \\
N & 19 \\
N & 3 \\
N & 11 \\
N & 21 \\
N & 10\end{array}$ & $\begin{array}{l}\text { Pasteurized sweet, in- } \\
\text { oculited with sisecifie } \\
\text { oremisms, held - } \\
\text { hours, neitralized, and } \\
\text { ripened }\end{array}$ & 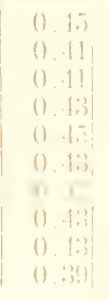 & 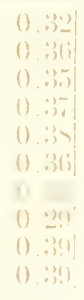 & & & & \\
\hline $\begin{array}{ll}\mathrm{R} & \mathrm{BI} \\
\mathrm{R} & 100 \\
\mathrm{R} & 2: 3 \\
\mathrm{R} & 12 \\
\mathrm{R} & 12 \\
\mathrm{R} & 18 \\
\mathrm{R} & 19 \\
\mathrm{R} & 8 \\
\mathrm{R} & 11 \\
\mathrm{~T} & 21 \\
\mathrm{R} & 210\end{array}$ & 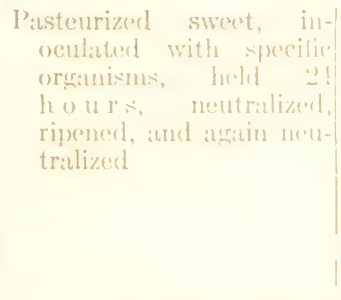 & 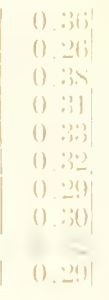 & 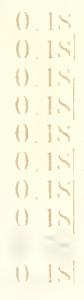 & Hishy & ()ily & $\begin{array}{l}\text { Fishy } \\
\ldots \ldots \ldots \\
\ldots \ldots \ldots \\
\ldots \ldots \\
\text { Itinncid } \\
\ldots \ldots \ldots\end{array}$ & $\begin{array}{l}\text { Fishy } \\
\text { Oily } \\
\ldots \ldots \ldots \\
\ldots \ldots \\
\text { Fishy } \\
\text { Wishy }\end{array}$ \\
\hline $\begin{array}{ll}X & B 1 \\
X & 100 \\
X & 23 \\
1 & 12 \\
X & 18 \\
X & 19 \\
X & 8 \\
X & 11 \\
1 & -1 \\
1 & 11\end{array}$ & $\begin{array}{l}\text { Pasteurized sweet, in- } \\
\text { oculisted with specilic } \\
\text { organisms, hald } 21 \\
\text { hosurs, and ripened }\end{array}$ & \begin{tabular}{l|}
$\cdots$ \\
$\cdots$ \\
$\cdots$
\end{tabular} & 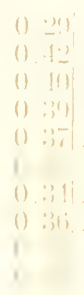 & $\begin{array}{l}\text { Xetollie } \\
\text { Metallice } \\
\text { Butyrie }\end{array}$ & $\begin{array}{l}\text { Mlotallice } \\
\text { Micotalice } \\
\text { Butyric }\end{array}$ & $\begin{array}{l}\text { 'Tithowy } \\
\text { lishy }\end{array}$ & $\begin{array}{l}\text { Irishy } \\
\text { Oily } \\
\text { Metallic } \\
\text { Strung } \\
\ldots \ldots \ldots \\
\ldots \ldots \ldots\end{array}$ \\
\hline
\end{tabular}

manifestation of these flavors is greatly mhanced by the growth of the

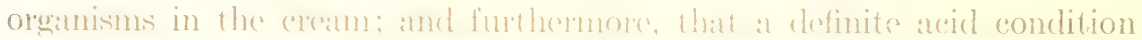
is essentiat for the development of these flatrons, which are potentially

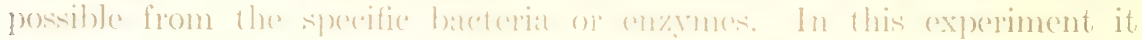
is appreciated that the results ate obtamed by an alsectative action with the orgamisms in the stater and these surviving pastenrization;

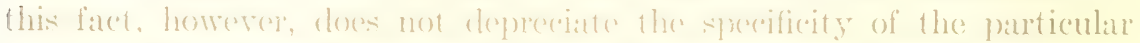
organisms that were inoculated. In reviewing the data from this experi- 
ment, it is interesting to note that the sweet pasteurized cream to which lactic acid had been added and which was uninoculated, developed the fishy flavor. The deseription of the flavor in the same sample of butter by different judges again calls attention to the fact that there seem to bo some conditions eommon to the fishy, the metallie, and the oily flavor.

The most consistent comments from specific organisms seem to be from cultures BI and 23. The former is Bact. ichthyosmizes, which wats obtained from Hammer; the litter is an organism isolated from rawripened-eream buter which dereloped the fishy flavor after two months and retained it for nearly twenty months.

Butter samples E 23 and X BI were analyzed for trimethylamine and ammonia. None of the former substance was found. Sample X 131 showed 35.2 parts per million of ammonia, and sample E 23 showed 17 parts per million.

\section{LONGEVITY OF BACTERIUM ICHTHYOSMUS' IN BUTTER}

Preliminary experiments with Bacterium ichthyosmius indicated that this organism might produce the fishy flavor in butter. It secmed dresirable, therefore, to determine its longevity in butter mate from the inoculated eream. Lesults of the guantitative deteminations of the bacteria in salted butter containing this organism are shown in table 2:); results are given only for those samples to which a chantateristic flavor was assigned.

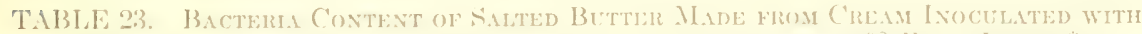

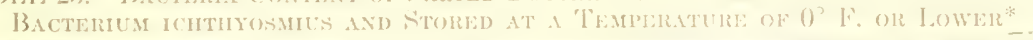

\begin{tabular}{|c|c|c|c|}
\hline \multirow{2}{*}{ Age of sumple (tays) } & \multicolumn{3}{|c|}{ Xumber of bapteria per gram } \\
\hline & K BI & IR BI & $\mathrm{IIBI}$ \\
\hline 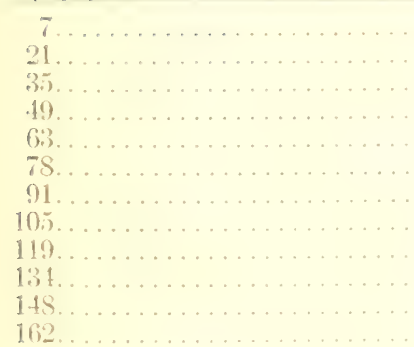 & 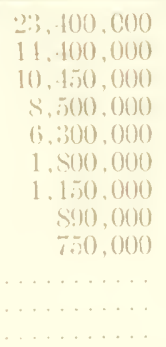 & 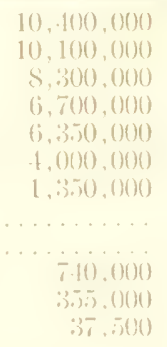 & 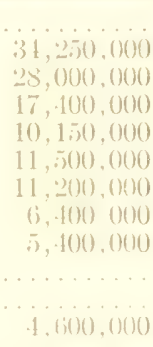 \\
\hline
\end{tabular}

* These results were furnished by J. T. Cusick. 
The deerease in bacterial content of the salted butter containing Bact. ichenyosmius show that, altho this organism may contribute to the development of the fishy flaror in butter, it does not do so by active multiplisation in that merlium.

FERTHER STIDIFS WITH BACTERIUM IOHTHYOSMIIUS

The results olotained with Bucterium ichthyosmius seemed to warrant a further study of its relationship to the fishy ftavor in butter. The following experiment was arried out with the puppose of determining the enenditions in butter under which the development of the flavor could be alereleraterl. Pastentrael sweet eream was inoculated with this oreanism and hedel for f wo disys at room temperature. The butter made from this eream was divided into twelve patrts, and to (ach of these parts a different substanere was alderl. The eream at the time of chuming contained (0.2:3 perp' cont acid. 'The treatmont of this butter', and the time of ocenrence of the finthy flavor ats determiner by two or more juderes, are shown in tathe 21 :

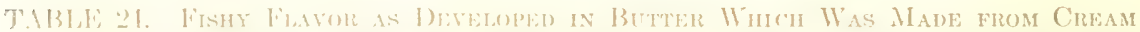

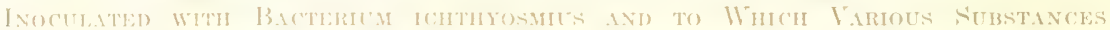

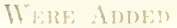

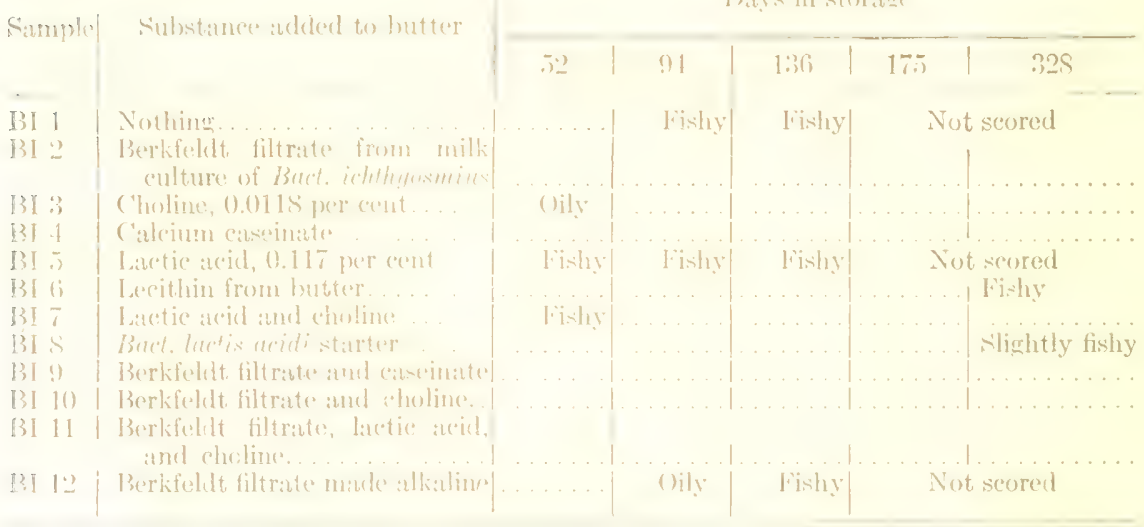

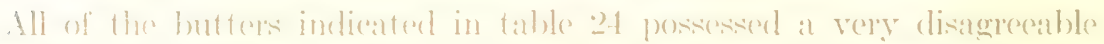

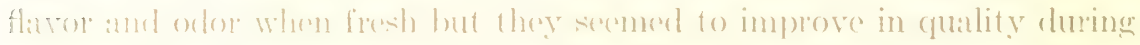

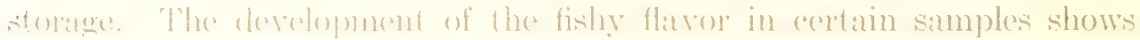
some ray interestibg leatures. In reviewing the results of this experi- 
ment it must be borne in mind that the eream from which they were made contained the pioducts of two disy growth of lanet. ichehyosmize.

The development of the fishy flavor in the sample to which nothing was adderl, is therefore signifieant. 'The carliex' ocemrence of the flavor in the sample to which laxetie ached was added is signifieant in that it confirms eertain obserrations already noted. The development of the flavor in the sample containimg the alkaline Berkfeldt filtrate seems to be about simultanerous with its clorelopment in the sample to which nothing was adterd. 'The lack of development of the flator in the samples to which the filtrate was adeded alone or in combination with other substanees, might appoar to be comblatictory to the enzymatic islea previously expresied. It, is believert, homereer, that this is more than offset by the othere data, which point te) the neersity of a definite set of eonditions that must be met in order fo produce the flatror. sueh being the case, the absenee of the flater when the filt wate mats adderl may be explained on the basis that the propere erpulitumim hat been disturbert. The final oceuremee of fishiness in the sample mettaning lerithin is of importaned as indieating that this may be the mether substanes of the material eausing the flatror. ()ther seattering results do not merit particular discussion at this time.

The trimethylanine and ammonia content of the samples shown in table 21, and their acid value, are given in table: 25:

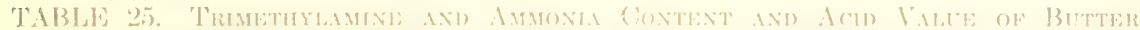

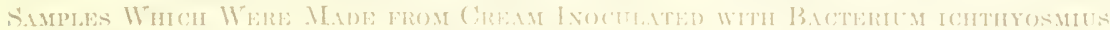

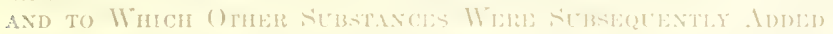

\section{Simmle:}

\section{Aer of
sample
(dizlat}

(c) matis yer million.

\section{$X I I_{3}$} (p)irts pere milliuns
Acid vithe

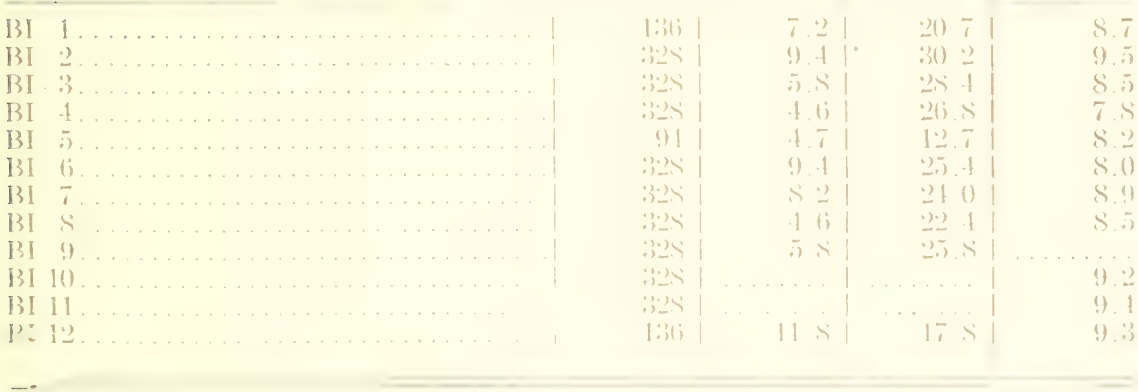


The relatively constant trimethylamine results shown in table 25 inclieate strongly that this substance hats been produed in the cream by Bect. ichthyosmius.

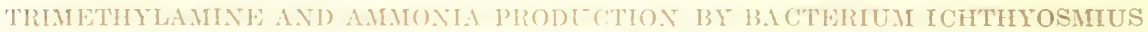
IX .HI.K A.NI) ('HE.MI

In order to deromine the trimethyimmine production by Bacterium

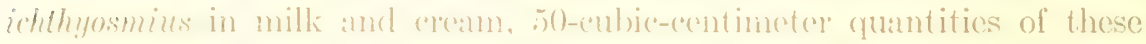
substaneses, sterilized, were inoculated with the organism alone and in rombination with a latefreacid stanter. The inoentations were held for forty hours at $330^{\circ}$ ('.. and the trimethylamine and ammonia were then

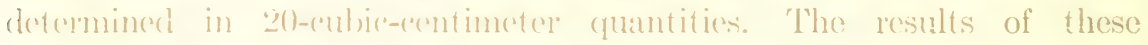
determinations are shown in talne 26 :

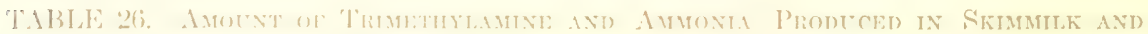

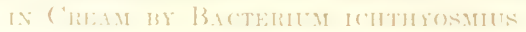

\begin{tabular}{|c|c|c|c|}
\hline Inoculation & Miaterial inoculated & $\begin{array}{l}\left(\mathrm{CH}_{3}\right)_{3} \mathrm{~T}^{\top} \\
\text { (parts per } \\
\text { miliou) }\end{array}$ & $\begin{array}{c}\mathrm{NII}_{3} \\
\text { (parts per } \\
\text { million) }\end{array}$ \\
\hline 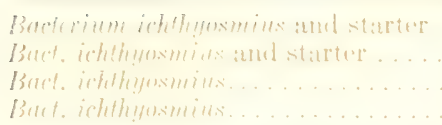 & $\begin{array}{l}\text { skimmilk. . . . . . } \\
\text { ('rum . . . . . . . . . . . . . } \\
\text { ('rmam . . . }\end{array}$ & $\begin{array}{l}\text { None } \\
204.0 \\
97.4 \\
74.7\end{array}$ & $\begin{array}{r}81 \\
85 \\
125 \\
78\end{array}$ \\
\hline
\end{tabular}

The results presentert in tathle 226 alde of ereat interest as showing beyond a doubt that the fishy flatror prexlueres in milk and eream by Bact.

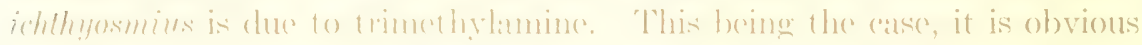
that this substanee woukt be carried into the butere and there, under propere conclitions which have atreaty heren printerteont, be responsible

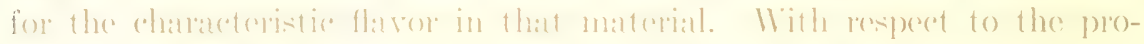
cluetion of trincthytamine in areann and in milk be this organism, it is desirable to again call attention to the olsecrations of the author, in which the erolution of a fishy flavor wats noted on the adelition of alkali to swret areamin.

These results atre of further importanee in that the cream inoculated with starter and Bact. Cehthyosmius contained at greater amount of trimethylanine tham did the cream inocelated with the oreatuism allone. This indieates that an ateid condition is most fitrorable for this particular 
fermentation, which would be in hamony with the idea that lecithin furnishes the soures of the trimethylamine produed by the oranism. The restilts are supported also by the fact that lowithin is largely associated with the fat, and that acenreling to Iammarsten and I Iextin (1915) lecithin is decomposed by dihute acids. Such being the ease, it is readily seen that this fermentation brought ahout by Boct. ichthyosmius would be greatly enhaneed by the presenes of acid. The presenes of trimethylamine in skimmilk inoesulated with the organism alone might be explained on the basis that the organism wats ables to proture this substance from proteins als weel! als from lecithin. Certain datal not included in this patper, howererr, indieate that theres is a cortain amount of lecithin present in skimmilk. Just why there is no trimethylamine in skimmilk inoenlated with the starter and the organism, is more diffient to explain. It may be that the greater acidity in the skimmilk has inhibited the particular factor responsible for trimethylamine production.

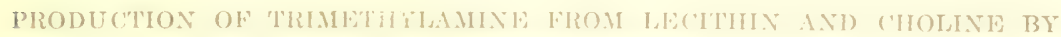

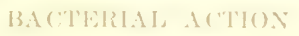

In order to determine, if possible, whether certain organisms found

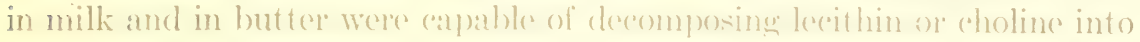
trimethylamine, a series of inoculation experiments were carried out. Lecithin alone in 0.5 pere cent concentration, and in the presenese of laetic acid and salt, was inoculated with a number of organisms, somes of which were obtained from mikk, some from fishy luttere and some from

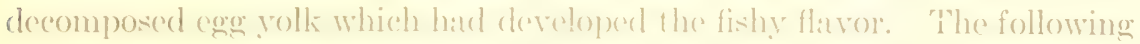
known speciss were also used: Bucterium lactis aridi. Bact. aerogenes, Bacillus prodigiosus, B. proteus, Bucterium ichthyesmius, P'sendomonus. liquefaciens fluoreseens, ()idium lactis. All organisms were inoculated singly and in various eombinations, and the eultures were held at $20^{\circ} \mathrm{C}$. for approximately nine monthes. At the and of that time the enltures were tested for the presenes of trincthylamine by heating with allati. Negative results were obtained from all of the lecithin inoeulations testerd. Unfortunately, many of the eultures were contaminated with mold, and, since the results could not be considered trust worthy, they were discateded.

The same series of experiments was repeaterl by inoculating ().1-percent choline alone and in the presenee of latetie aleid and salt. These cultures were held under the same conditions ats were the lecithin inoenlat- 
tions. Trimothylamino mas found where Bucterium tebthyosmius was inoculated alone, in combintion with f) tium lactis, and with Bucterium lactis acidi. The presones of sill did not serm to prevent the production of trimethylamine. Two organisme which were isolated from milk gave a pronounced test from the choline inoulation, hut gitre neative results

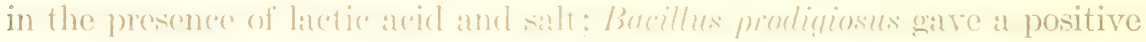
reaction from the choline alone; and bucterimm actorenes gave a non-

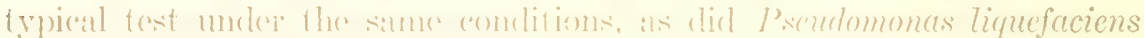

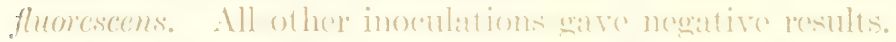

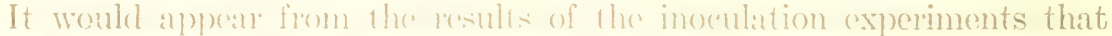

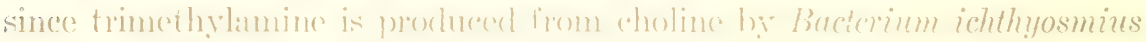

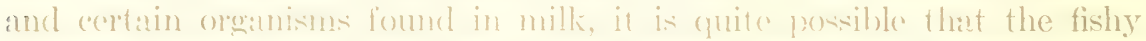

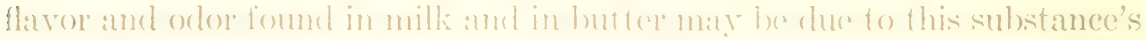

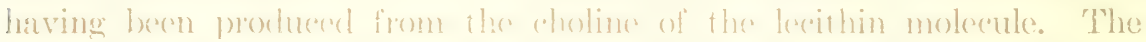

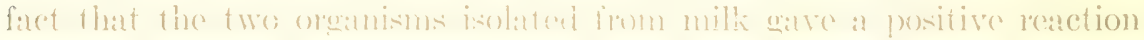

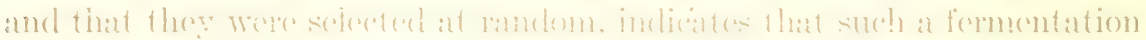

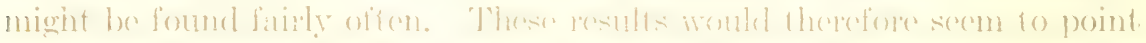

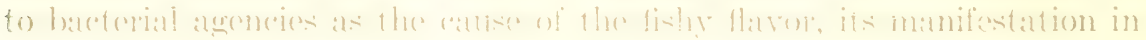

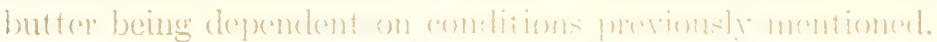

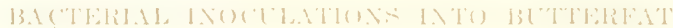

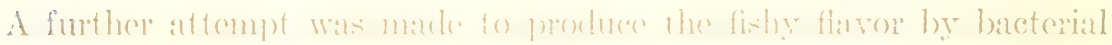

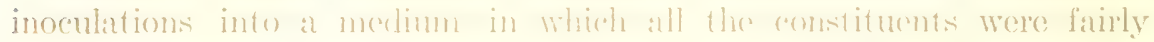

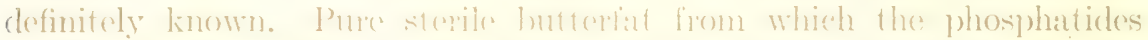

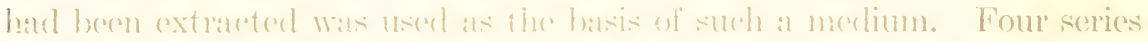

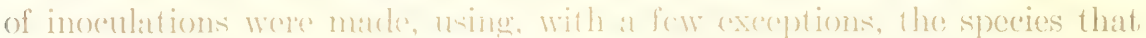

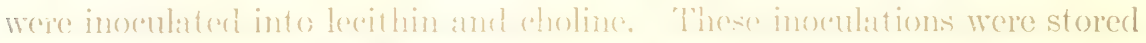

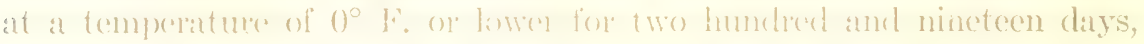
at the ond of which time they were eximment for the fishy flaror by four

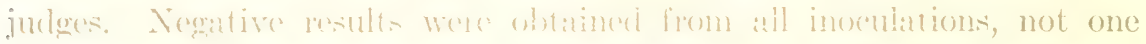

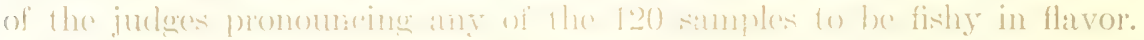

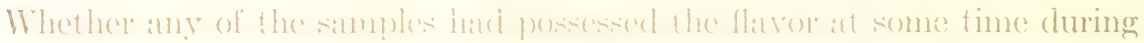

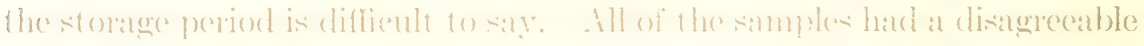

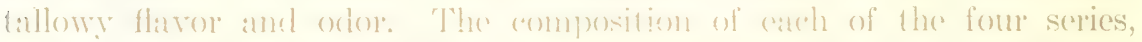

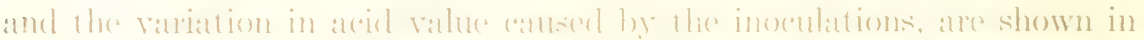

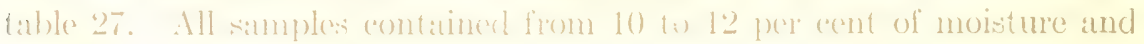
$2 p x+c$ cont of sill. 


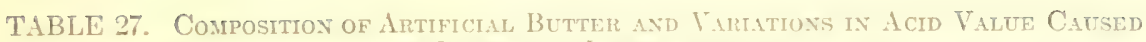
BY BACTERLAI. INOCULATIONS

\begin{tabular}{|c|c|c|c|c|c|}
\hline \multirow[b]{2}{*}{$\begin{array}{l}\text { Sam- } \\
\text { ple }\end{array}$} & \multirow[b]{2}{*}{ Organism or source } & \multicolumn{4}{|c|}{ Composition and acid value } \\
\hline & & $\begin{array}{l}\text { Butter- } \\
\text { fat }\end{array}$ & $\begin{array}{l}\text { Butterfat } \\
\text { and } 0.15 \\
\text { per cent } \\
\text { of lecithin }\end{array}$ & $\begin{array}{l}\text { Butterfat } \\
\text { and } \\
\text { IBerkfoldt } \\
\text { fi!trate from } \\
\text { starter }\end{array}$ & $\begin{array}{l}\text { Butterfat, } \\
\text { Berkfeldt } \\
\text { filtrate from } \\
\text { stitrter, and } \\
0.1 \text { is per cent } \\
\text { of lecithin. }\end{array}$ \\
\hline $\begin{array}{r}1 \\
2 \\
3 \\
4 \\
5 \\
6 \\
7 \\
3 \\
9 \\
10 \\
11 \\
12 \\
13 \\
14 \\
15 \\
16 \\
17 \\
18 \\
19 \\
20 \\
21 \\
22 \\
23 \\
21 \\
25 \\
26 \\
27 \\
28 \\
29 \\
30\end{array}$ & 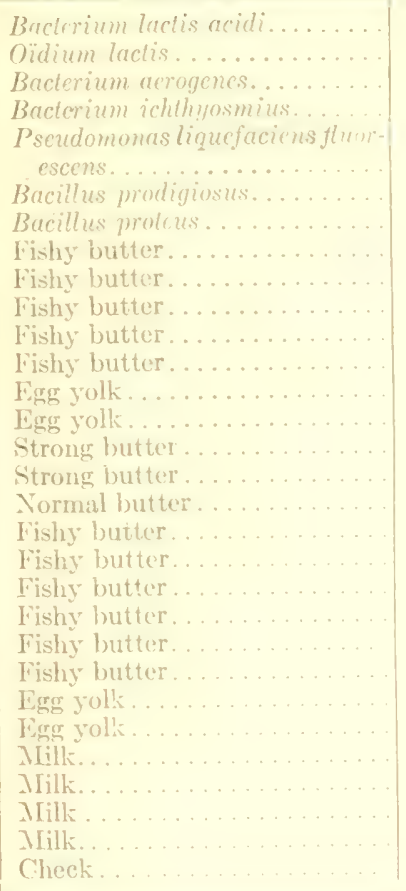 & 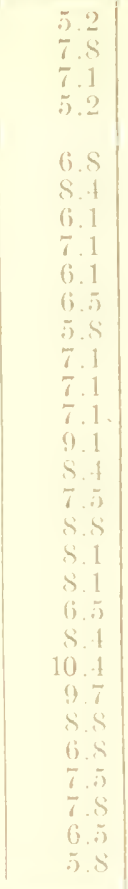 & 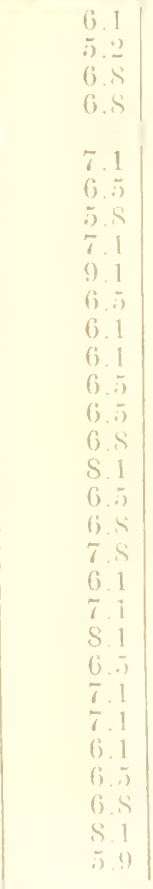 & $\begin{array}{l}6.5 \\
7.8 \\
5.8 \\
6.1 \\
7.1 \\
7.5 \\
6.5 \\
7.5 \\
6.5 \\
7.8 \\
7.8 \\
8.8 \\
8.1 \\
8.37 \\
8.1 \\
8.1 \\
8.4 \\
8.1 \\
7.5 \\
8.1 \\
8.1 \\
9.4 \\
8.1 \\
8.1 \\
6.5 \\
7.5 \\
7.1 \\
6.1 \\
8.1 \\
5.1\end{array}$ & $\begin{array}{r}8.8 \\
7.5 \\
7.8 \\
7.1 \\
7.1 \\
7.1 \\
6.8 \\
7.8 \\
7.5 \\
7.1 \\
8.8 \\
8.1 \\
8.1 \\
9.7 \\
7.8 \\
8.4 \\
8.4 \\
8.8 \\
9.7 \\
10.1 \\
11.0 \\
7.8 \\
7.8 \\
7.1 \\
7.8 \\
7.8 \\
9.1 \\
10.0 \\
5.8 \\
7.4\end{array}$ \\
\hline
\end{tabular}

The results shown in table 27 ane of interest only to the extent that they show the variation in aciel value eatused by different sluecies of bacteria. Inasmuch as the samples were placed in storage immediately after being inoculated, it is probable that the chamgist atre the result of bacterial enzymes liberated by antolysis, herause it hats been repentedly shown that litile or no growth takes place during storage. 


\section{SUMIIARY}

The data presented in this paper show beyond a doubt that there is in nomml butter a sufficient amount of lecithin to yield, on decomposition, small (uantities of trimethylamine, and it is shown also that small quantities of this substance are essential for the manifestation of a fishy odor. Furthermore, it is shown that when this substance is worked into Dutter under the proper conditions, it produees a flavor deseribed as fishy. These results are most uniform when trimethylamine butrate is usecl. An issociative fermentation in butter or in cream, with the ultimate formation of this substance, is quite possible. As to whether or not this or some of her volatile and unstable combination of trimethylamine is the eatuse of the natural fishy flatror, remains to be shown more eonchisively. (ertain data do indieate that trimethylamine is found in some samples of fishy-flarored butter but not in others. Altho it is posible that its presener is incidental in such samples, that is not believerd to be the rase. In this eonnection it is worth while to call attention to the confusion betwern the fishy, oily, and metallie flavors when they are present to only a slight dewere. It secmes possible that the initiation of the development of these flatrors depends on a common fundamental factor. Whether or not any particular one of them derelops to its typieal flatron would depend on the presence of certain conditions which were speceifie for that flatror. With this possibility in view, it would be logical to assume that trimethylamine is responsible for the typeal herring, or

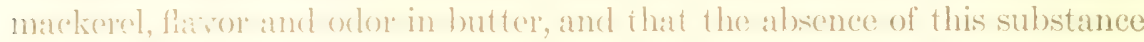
would result in the manifestation of similar but non-typieal flatvors.

There serems to be no doubt that the presenee of a definite acid condition in the butter is esential for the development of the fishy flavor. This eomdition is best obtained when butter is made from eream confaining lactic acid, regandless of whether this is developed by bacteria or atderl to the eream in the form of the commereial product. Furthermore, the results inclicate that, while a definite acid condition is essential, it must be alecompanied by some other equally important factor. 'The data show that this factor is determined by biological agencies. It appears that both these factors must exist in a definite and delicate relationship, and that if the propere ecpuilibrium is disturbed, the characteristic flavor is not manifest. Nimnerous results and observations indicate that the unknown tiansient factor is trimethylamine. 
The bacteriologieal aspects of the problem secm to involve the determination of the relationship already mentioned. It is shown that the acid value of butter is to a certain extent regulated by biological factors. probably enzymes. It is shown also that trimethylamine may be produced in milk and in cream, probably to some extent from lecithin, with the consequent production of the fishy flavor in those products. Furthermore, it is shown that Bacterium ichthyosmius, which produced the flaror in those substanees, would produere the flavor in butter also undere certain conditions. It would therefore seem posible that other species of microorganisms might bring about the same type of change. It seems highly proballe that the growth of bactoria in the cream before it is made into butter determines the conditions necessary for the later manifestation of the fishy fiavor.

The data dealing with leceithin ats the souree of trimethylanine in milk products are too meager to warrant definite conclusions at this time. However, the results presented herein, taken together with what is known regarding this substance, indicate that this is one of the most logical sources.

\section{ACKYOWIEDGMIENTS}

The author wishes to express his lieen appreciation and gratitude to Dr. E. S. Guthrie, Mr. W. L. Ayres, and Mr. H. C. Jackson, of the Department of Dairy Industry at Comell Lniversity, to all other nembers of this Department who have been kind cnough to aid in the scoring of butter, and to all who have furnished simmples for study. 


\section{BIBLIOGiRAPHY}

Bafierr, Cienrge. 'The simpler' natural bases, p. 1-215. (Reference on p. 12.) 1914

Bordas, F., and Raczowsin, Sic, De. Variation de l'acide phosphorique suivant l'ầge du lait. Acad. Sei. [Paris]. Compt. rend. I35:302-303. 1902.

Browve, C. A., JP. A contribution to the chemistry of butter-fat. II. 'The chemicul composition of butter-fat. Amer. Chem. Soc. Journ. $2 \mathrm{r}: 807-827 . \quad 1899$

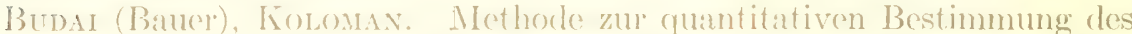
Ammoniaks und Trimethylamins. K/stehr. physiol. Chem. 86:107121. 1913.

DAvIs, IT. A. Annines and ammonium hases. In Allen's commercial organic antlyis 6:1-19). (Reference om p, 16.) 1912.

FETZER, L. W. The leceithin (ontent of nilk under pathologic conditions. Abstracted in seienee H. $8.33: 339.91911$.

Folix, ()tom, and Machlum, A. B. ()n the determination of ammonia in unine. Jount biol. (heme. II:52:3-525). 1912.

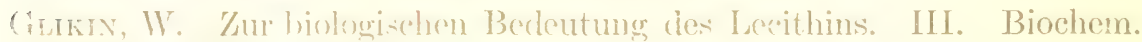
Zischr. 2I:248. 1900!)

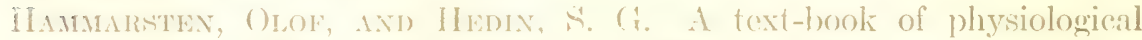
ahemistry. 7therl. (Roforemee on p. 24:3.) 1915.

IInmmer, B. W. Fishome in evamorated milk. Iowa Agr. Ixp). Sta.

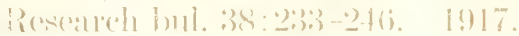

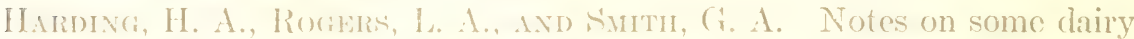
troubles. New lork ((rencra) Aer. Fxy). Sta. Bul. 183:173-193. (Referenee on p. 181.) 1900.

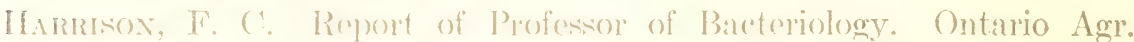
('oll. and Lxp. Fam. Ann. rep) $27(1901): 7.4-81$. (Reference on p. 79.) $19(1) 2$.

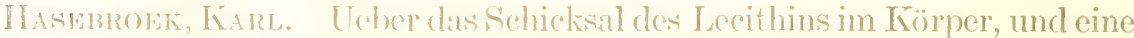
Boyichung (lesselben zum Sumpfgas im Darmeanal. Zischr. physiol.

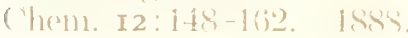

Hexzкек, (). F. Investigations in dairy manufactures. In Report of the I martment of Thirr Husbandry. Purdue Univ. Agr. Exp. Sta. All11. rept. $28(1915): 39 \% 1916$. 
Koch, Wazdexar. Die Lecithane und ihre Berdentung für die lebende Zelle. Zitschr. physiol. Chem. 37:181-188. 1902-03,

Leathes, J. B. The fats, p. 1-13.S. (Reference on p. 45.) 1913.

Loxa, J. H. Observations on the stability of lecithin. Amer. Chem. Soc. Joum. 30:881-895. 1908.

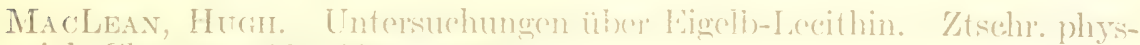
iol. Chem. $59: 22: 3-229.1909$.

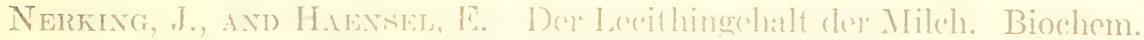
Ztschr. I3:348-35:3. 19708.

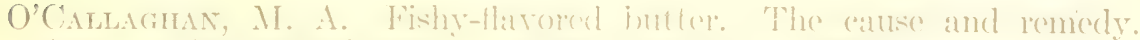

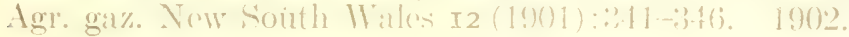

Butter classifention. The scicutifie examination of butter for export to Englund. Agr. $291 \%$. Now sonth llales I8 $(1907): 22: 3-227$. 1908.

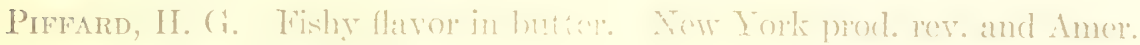
(reamery $132: 20 . \quad 1901$.

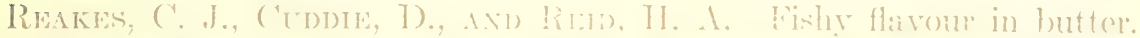
A preliminary note on an ingatimy inte the cattese of the defeet. Journ. New Zealund 1)ept. Agr. 4:1-6i. 1912.

Rociers, I. A. Fishy flavor in houter. I'. S. Bur. Anim. Indus. Cir. $14(0: 1-20.190()$.

'The dexelopment of fishy flavol in hutter. Wisensin Buttermakers dssn. Proe. I3:7(). 191.t a.

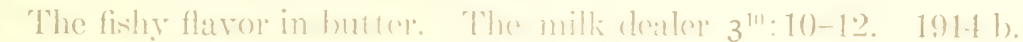

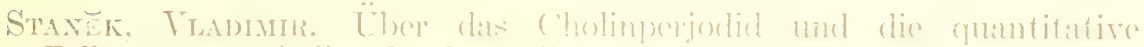

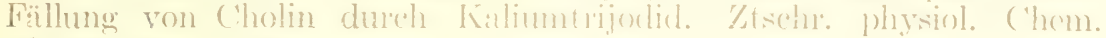
$46: 28(0-28 \tilde{r} .19(0)$.

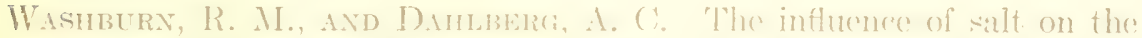
changes taking place in storage butter. fourn. dairy sei. I:114-126. 1918 .

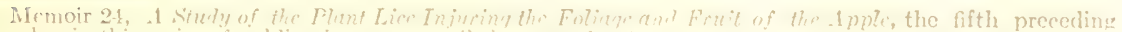

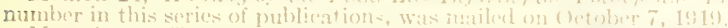

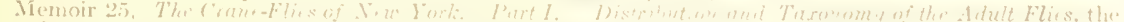

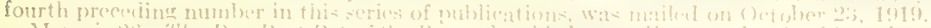

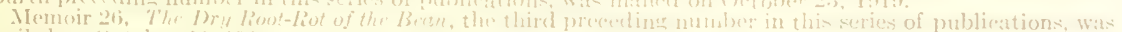
mailed on (Oetober 23,1919 



